Journal for ImmunoTherapy of Cancer

\title{
Upfront dose-reduced chemotherapy synergizes with immunotherapy to optimize chemoimmunotherapy in squamous cell lung carcinoma
}

\author{
Xiran He, ${ }^{1,2}$ Yang Du, ${ }^{2,3}$ Zhijie Wang, ${ }^{1}$ Xin Wang, ${ }^{1}$ Jianchun Duan, ${ }^{1}$ Rui Wan, ${ }^{1}$ \\ Jiachen Xu, ${ }^{1}$ Pei Zhang, ${ }^{1,2}$ Di Wang, ${ }^{1,2}$ Yanhua Tian, ${ }^{4}$ Jiefei Han, ${ }^{1}$ Kailun Fei, ${ }^{1}$ \\ Hua Bai, ${ }^{1}$ Jie Tian, ${ }^{2,5}$ Jie Wang (1) ${ }^{1}$
}

To cite: He X, Du Y, Wang Z, et al. Upfront dose-reduced chemotherapy synergizes with immunotherapy to optimize chemoimmunotherapy in squamous cell lung carcinoma. Journal for ImmunoTherapy of Cancer 2020;8: 0000807. doi:10.1136/jitc-2020-000807

- Additional material is published online only. To view please visit the journal online (http://dx.doi.org/10.1136/jitc2020-000807).

$\mathrm{XH}$ and YD contributed equally.

Accepted 29 September 2020

Check for updates

(C) Author(s) (or their employer(s)) 2020. Re-use permitted under CC BY-NC. No commercial re-use. See rights and permissions. Published by BMJ.

For numbered affiliations see end of article.

Correspondence to Dr Jie Wang; zlhuxi@163.com

Dr Jie Tian; jie.tian@ia.ac.cn

Dr Hua Bai; baihuahb@sina.com

\section{ABSTRACT}

Background The survival benefits of combining chemotherapy (at the maximum tolerated dose, MTD) with concurrent immunotherapy, collectively referred to as chemoimmunotherapy, for the treatment of squamous cell lung carcinoma (SQCLC) have been confirmed in recent clinical trials. Nevertheless, optimization of chemoimmunotherapy in order to enhance the efficacy of immune checkpoint inhibitors (ICls) in SQCLC remains to be explored.

Methods Cell lines, syngeneic immunocompetent mouse models, and patients' peripheral blood mononuclear cells were used in order to comprehensively explore how to enhance ectopic lymphoid-like structures (ELSs) and upregulate the therapeutic targets of anti-programmed death 1 (PD-1)/anti-PD-1 ligand (PD-L1) monoclonal antibodies (mAbs), thus rendering SQCLC more sensitive to ICls. In addition, molecular mechanisms underlying optimization were characterized.

Results Low-dose chemotherapy contributed to an enhanced antigen exposure via the phosphatidylinositol 3-kinase/Akt/transcription factor nuclear factor kappa B signaling pathway. Improved antigen uptake and presentation by activated dendritic cells (DCs) was observed, thus invoking specific T cell responses leading to systemic immune responses and immunological memory. In turn, enhanced antitumor ELSs and PD-1/ PD-L1 expression was observed in vivo. Moreover, upfront metronomic (low-dose and frequent administration) chemotherapy extended the time window of the immunostimulatory effect and effectively synergized with anti-PD-1/PD-L1 mAbs. A possible mechanism underlying this synergy is the increase of activated type I macrophages, DCs, and cytotoxic $\mathrm{CD}^{+} \mathrm{T}$ cells, as well as the maintenance of intestinal gut microbiota diversity and composition. In contrast, when combining routine MTD chemotherapy with ICls, the effects appeared to be additive rather than synergistic.

Conclusions We first attempted to optimize chemoimmunotherapy for SQCLC by investigating different combinatorial modes. Compared with the MTD chemotherapy used in current clinical practice, upfront metronomic chemotherapy performed better with subsequent anti-PD-1/PD-L1 mAb treatment. This combination approach is worth investigating in other types of tumors, followed by translation into the clinic in the future.

\section{INTRODUCTION}

Squamous cell lung carcinoma (SQCLC) is a common subtype that accounts for $20 \%-30 \%$ of non-small cell lung cancer (NSCLC) cases. ${ }^{1}$ Over the past few decades, there have been limited advances in SQCLC treatment owing to a lack of targetable mutations and low immunogenicity. SQCLC can be classified as a 'cold' tumor, meaning it is less sensitive to immunotherapy. Platinum-based doublet chemotherapy at the routine maximum tolerated dose (MTD) has long been used as firstline care. ${ }^{2}{ }^{3}$ However, at the MTD, modest chemotherapeutic effects and substantial systemic toxicity make SQCLC treatment challenging. ${ }^{4}$

Recently, immune checkpoint inhibitors (ICIs), such as programmed death 1 (PD-1) and its ligand PD-L1, have revolutionized cancer therapy, particularly through their combination with chemotherapy. ${ }^{6} 7$ Such combination approaches are referred to as chemoimmunotherapy, and have been shown to significantly improve response rates when compared with ICI monotherapy. ${ }^{8}$ In the phase III KEYNOTE-407 and IMpower-131 trials conducted in patients with SQCLC, it was observed that pembrolizumab or atezolizumab combined with concurrent MTD chemotherapy resulted in greater clinical benefits with manageable toxicity when compared with chemotherapy plus placebo. ${ }^{910}$ Hence, it has been suggested that chemoimmunotherapy may be an excellent therapeutic strategy for patients with SQCLC. Nevertheless, despite the encouraging results obtained from these phase III clinical trials, the actual relationship between MTD chemotherapy 
and ICIs remains controversial. For the past few decades, most oncologists believed that MTD chemotherapeutics cause immunosuppression, including myelopenia or lymphopenia, suggestive of a possible antagonistic effect between MTD chemotherapy and immunotherapy. ${ }^{11-13}$ In addition, the toxicity of MTD chemotherapy in combination with concurrent ICIs should not be overlooked, especially for older or weak patients who might poorly tolerate this combination. It should be noted that older patients account for the majority of individuals with lung cancer, and have higher mortality rates compared with younger patients. ${ }^{1415}$ However, older patients are underrepresented in current standardized clinical trials, mainly due to functional disabilities and pre-existing comorbidities. ${ }^{16}$ Hence, more efficacious combinatorial strategies that can ultimately boost chemoimmunotherapy and ameliorate its adverse effects in patients with SQCLC are still worth exploring.

The immunoregulatory effects of low-dose chemotherapeutic drugs have been of particular interest within the field of oncology. ${ }^{17-22}$ In genetically engineered lung adenocarcinoma mouse models, Pfirschke et al observed that low-dose oxaliplatin (OxP) combined with cyclophosphamide triggered immunogenic responses and provided benefits when combined with ICIs. ${ }^{23}$ Similarly, Song et al demonstrated that low-dose OxP enhanced antitumor ectopic lymphoid-like structures (ELSs) in murine colorectal cancer models, and OxP combined with an anti-PD-L1 monoclonal antibody $(\mathrm{mAb})$ significantly inhibited tumor growth. ${ }^{24}$ Moreover, low-dose and frequent (so-called 'metronomic') administration of cyclophosphamide could extend the time window of immune modulation. ${ }^{25}{ }^{26}$ In another study, Liu et al found that the administration sequence and dosage of chemotherapeutic drugs and an anti-CD47 mAb significantly affected the host immune response following immunotherapy. ${ }^{27}$ Therefore, it was suggested that the administration dosage, frequency, and sequence of chemotherapeutic drug treatments are critical for potent immune activation, especially when combined with ICIs.

A functional and active immune system is pivotal for durable clinical responses, especially for responses to immunotherapy ${ }^{28}$ In order to achieve an optimal therapeutic effect through chemoimmunotherapy, chemotherapy should be considered as an initiator or partner of immunotherapy rather than as serving the classical role of a cytotoxic agent at the MTD, the sole purpose of which is to inhibit tumor growth. Hence, finding the balance between active antitumor immunity and tumor inhibition with less toxicity is critical for the success of chemoimmunotherapy. In this study, we first attempted to elucidate the underlying molecular mechanisms responsible for the synergistic effect between low-dose chemotherapy and immunotherapy in SQCLC in order to facilitate the design of more effective combinatorial chemoimmunotherapeutic approaches. Second, in order to achieve the balance between chemotherapy-induced immunosuppression and an active tumor-immune microenvironment
(TIME), different combinatorial chemoimmunotherapy regimens in terms of chemotherapy dosage, frequency, and administration sequence were further explored in SQCLC mouse models.

\section{MATERIALS AND METHODS \\ Reagents and antibodies}

Cisplatin (CDDP, Cat\# S1166), gemcitabine (GEM, Cat\# S1714), docetaxel (DTX, Cat\# S1148), paclitaxel (PTX, Cat\# S1150), LY $294002(\mathrm{PI} 3 \mathrm{Ka} / \delta / \beta$ inhibitor, Cat\# S1105), MK-2206 2HCl (Akt1/2/3 inhibitor, Cat\# S1078), and BAY 11-7082 (transcription factor nuclear factor kappa B/NF-кB inhibitor, Cat\# S2913) were purchased from Selleck Chemicals (Houston, Texas, USA). For in vitro experiments, these reagents were dissolved in sterile phosphate-buffered saline (PBS) or dimethyl sulfoxide (DMSO; final DMSO concentrations $<0.1 \%$ ) and stored at $-20^{\circ} \mathrm{C}$. For in vivo studies, solutions of the chemotherapeutic agents were prepared according to the manufacturer's specifications immediately prior to administration. Fetal bovine serum (FBS, Cat\# 12662029), penicillin-streptomycin (Cat\# 15140122), Glasgow's buffered minimal essential medium (Cat\# 21710082), RPMI1640 culture medium (Cat\# 61870044), and Dulbecco's modified Eagle's medium (DMEM, Cat\# 11965092) were purchased from Gibco (Grand Island, New York, USA). Human and murine recombinant interferon- $\gamma$ (IFN- $\gamma$ ) (Cat\# 713906 and Cat\# 714006), LEGENDplex recombinant granulocyte-macrophage colony-stimulating factor (GM-CSF, Cat\# 713704), recombinant interleukin-4 (IL-4, Cat\# 715004), and recombinant high mobility group box-1 (HMGB-1, Cat\# 764004) were purchased from Biolegend (Carlsbad, California, USA). Lipopolysaccharide solution (500 X) (Cat\# 00-4976-93) was purchased from eBioscience (Affymetrix, Thermo Fisher Scientific, San Diego, California, USA). Collagenase type IV (Cat\# C5138) and deoxyribonuclease I (Cat\# D4263) were purchased from Sigma-Aldrich Company (St. Louis, Missouri, USA) and were used to prepare singlecell suspensions from tumor tissue. All fluorescently conjugated antibodies used for fluorescence-activated cell sorting (FACS) were purchased from BD Biosciences (Oxford, UK). Murine and human commercial HMGB-1 ELISA kits (Cat\# HH0016 and MH0016) were obtained from NeoScientific (Cambridge, Massachusetts, USA). Clodronate, neutral clodronate liposomes (Cat\# F70101C-N), used for macrophage depletion, were purchased from FormuMax Scientific (Silicon Valley, California, USA). Anti-CD8 $\alpha$ mAb (clone 169.4, Cat\# BE0117) for $\mathrm{CD}^{+}{ }^{\mathrm{T}}$ cell depletion, anti-PD-1 mAb (CD279, clone 29F.1A12, Cat\# BP0273), anti-PD-L1 mAb (B7-H1, clone 10F.9G2, Cat\# BE0101), InVivoPure $\mathrm{pH}$ 7.0 dilution buffer (Cat\# IP0070), and InVivoPure $\mathrm{pH}$ 6.5 dilution buffer (Cat\# IP0065) were purchased from BioXcell (West Lebanon, New Hampshire, USA). 


\section{Cell lines and culture conditions}

The murine KLN-205 SQCLC cell line and human SQCLC cell lines SK-MES, H226, and H520 were obtained from the American Type Culture Collection (Manassas, Virginia, USA). OVA antigen-expressing KLN-205 cells were obtained from COBOIER Biotechnology Co. (Nanjing, China). These cells were cultured in Glasgow's buffered minimal essential medium or RPMI-1640 culture medium, supplemented with $15 \%$ FBS and $1 \%$ penicillin-streptomycin in a humidified incubator with $5 \% \mathrm{CO}_{2}$ at $37^{\circ} \mathrm{C}$. For bioluminescence imaging (BLI) in vivo, we transfected wild-type KLN-205 cells with a luciferase (Luc) gene/lentivirus in order to establish KLN-205-Luc cells following the manufacturer's protocol (GeneCopoeia, Rockville, Maryland, USA). We confirmed that the proliferation, apoptosis, and differentiation of KLN-205-Luc cells were comparable to those of wild-type KLN-205 cells. In order to establish murine immature bone marrow-derived dendritic cells (BMDCs), we extracted bone marrow from the femurs and tibias of DBA-2J mice and incubated it with lysis buffer for 4 min in order to lyse red blood cells (RBCs). After rinsing with cell culture medium, we seeded $4 \times 10^{5}$ cells per well into 6-well plates and cultured them in $2 \mathrm{~mL}$ of DMEM containing 10\% FBS, $20 \mathrm{ng} / \mathrm{mL}$ GM-CSF, and $10 \mathrm{ng} / \mathrm{mL}$ IL-4. Fifty per cent of the medium was replaced with fresh culture medium every 2 days. After 5 days, we harvested non-adherent and loosely adherent cells, and used them for BMDC experiments.

\section{Establishment of SQCLC syngeneic mouse models and BLI in vivo}

DBA-2J male mice (7-8 weeks old, 21-23 g) were purchased from Beijing HFK Bioscience Co. (Beijing, China). We implanted $5 \times 10^{5} \mathrm{KLN} 205$-Luc cells into the right flanks of mice by subcutaneous injection. When the tumors were palpable, mice were randomly divided into different treatment groups. BLI was used to dynamically monitor tumor growth in vivo. Mice were anesthetized with $3 \%$ isoflurane and were then intraperitoneally injected with D-luciferin $(120 \mathrm{mg} / \mathrm{kg}$ in $100 \mu \mathrm{L})$. After 4 min, we used the Caliper IVIS Lumina III Live Imaging System to evaluate Luc light emission. After treatment, tumors and spleens were harvested for further evaluation.

\section{Fluorescence-activated cell sorting}

Cells grown after drug treatment or incubation with conditioned media were harvested and rinsed once with prechilled PBS. Cells were subsequently stained with an anti-CD16/CD32 antibody for $30 \mathrm{~min}$ at room temperature (RT) to block Fc receptors, and were then labeled with the appropriate fluorescein isothiocyanateconjugated antibodies for $30 \mathrm{~min}$ at $4^{\circ} \mathrm{C}$, followed by washing once with PBS. To prepare single-cell tumor tissue suspensions for FACS, tumors were digested in FBSfree RPMI-1640 culture medium containing $1 \mathrm{mg} / \mathrm{mL}$ collagenase type IV and $1 \mu \mathrm{g} / \mathrm{mL}$ deoxyribonuclease I at $37^{\circ} \mathrm{C}$ for $90 \mathrm{~min}$ and were then filtered through $100 \mu \mathrm{m}$ cell strainers (Corning Incorporated, Corning, New York, USA). To prepare single-cell spleen tissue suspensions, spleens were crushed with tweezers, filtered through cell strainers, and incubated with RBC lysis buffer at RT for 5 min. The KLN 205-Luc cells were frozen and thawed three times, and the resulting cell debris was used to stimulate the single-cell spleen suspensions overnight. The subsequent steps were the same as described for cell lines. We analyzed the cells with a FACScan cytometer (Becton Dickinson) and acquired 10,000 or 100,000 cells for each sample.

\section{ELISA}

To assess HMGB-1 secreted into the media, we treated cells (as described above) and collected the conditioned medium (supernatant). After centrifugation at 3500 rpm for 5 min, we assessed HMGB-1 in the conditioned medium by ELISA, following the kit manufacturer's instructions.

\section{Statistical analysis}

Quantitative data represent the mean \pm SD. SPSS software (V.16.0) was used to analyze the data, and GraphPad Prism V.5.0 (La Jolla, California, USA) was used to create the graphs. A Student's t-test or a Mann-Whitney U test was used to evaluate statistical significance, with $\mathrm{p}<0.05$ considered statistically significant (no significance; $\left.{ }^{*} \mathrm{p}<0.05 ; * \mathrm{p}<0.01 ; * * \mathrm{p}<0.001\right)$.

\section{RESULTS}

\section{Low-dose chemotherapy induces immunogenic cell death via the phosphatidylinositol 3-kinase/Akt/NF-кB signaling pathway in SQCLC}

Immunogenic cell death (ICD) activates the host immune system and enhances antitumor immune responses. During ICD, cancer cell antigens, including neoantigens, may be released and recognized thereby triggering greater immune responses. ${ }^{29}$ Primary ICD markers include major histocompatibility complex (MHC)-class I, human leukocyte antigen (HLA)-A/B/C, and HMGB-1. The cytotoxic effects of chemotherapeutic drugs in different SQCLC cell lines were evaluated using the Cell Counting Kit- 8 , and the $25 \%$ and $50 \%$ maximal inhibitory concentrations $\left(\mathrm{IC}_{25}\right.$ and $\left.\mathrm{IC}_{50}\right)$ were calculated using the SPSS V.16.0 software and were used in subsequent experiments (online supplemental figure S1). As shown in online supplemental figure S2, low-dose chemotherapeutic agents (CDDP, GEM, DTX, PTX) upregulated ICD markers and PD-L1 expression in a dose-dependent and time-dependent manner, thereby indicating increased (neo)antigen exposure and enhanced SQCLC immunogenicity. High-throughput RNA sequencing was then performed to identify the signaling pathways potentially involved in ICD induction (figure 1A, total landscape). The data revealed that 178 immunity-related genes were significantly differentially expressed in KLN-205 cells after treatment with low-dose chemotherapeutic agents 


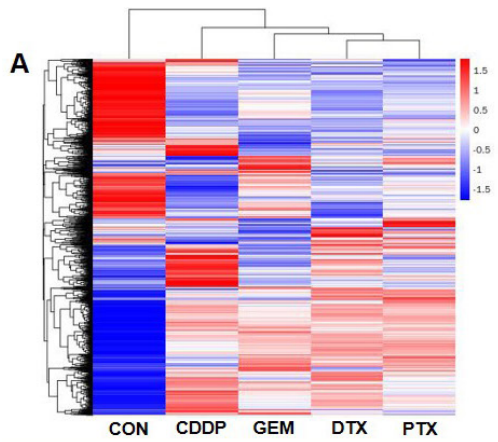

B
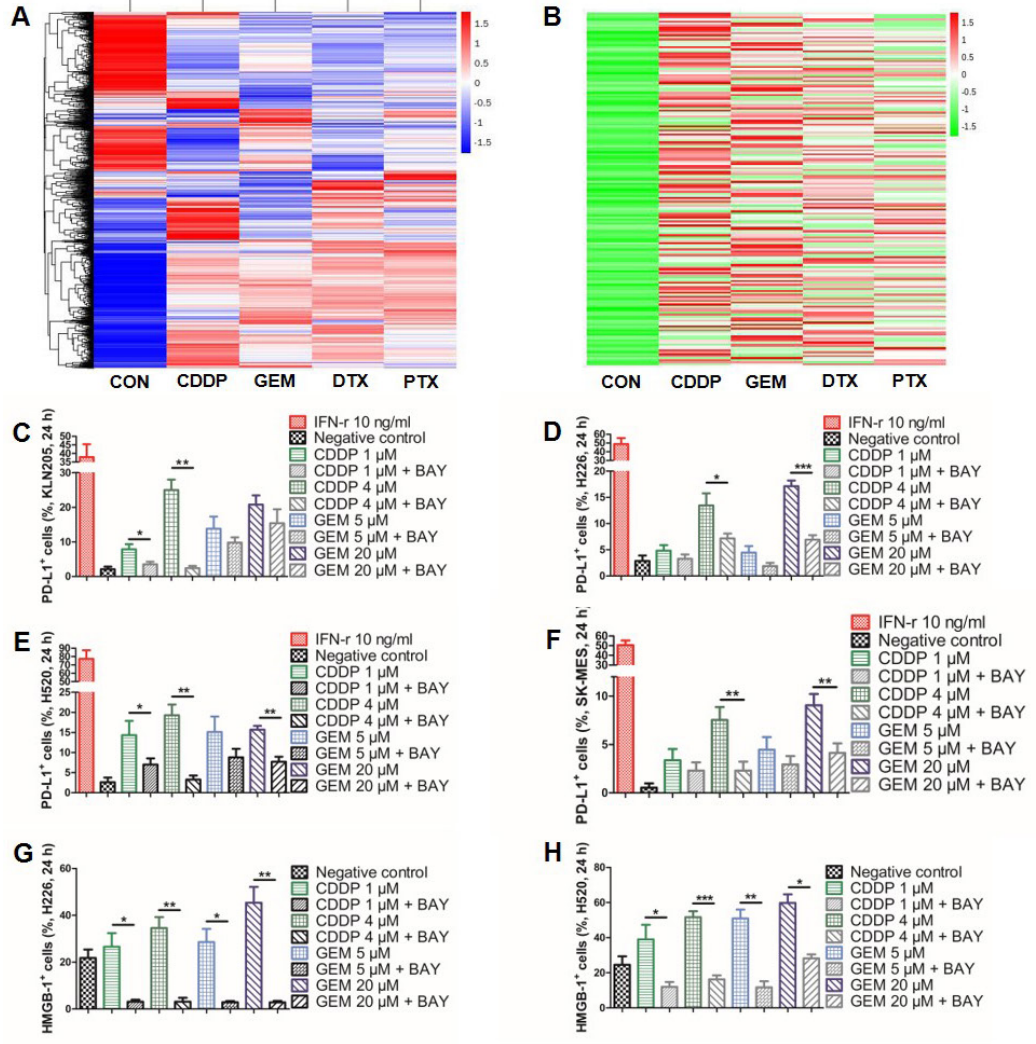

$H \equiv$
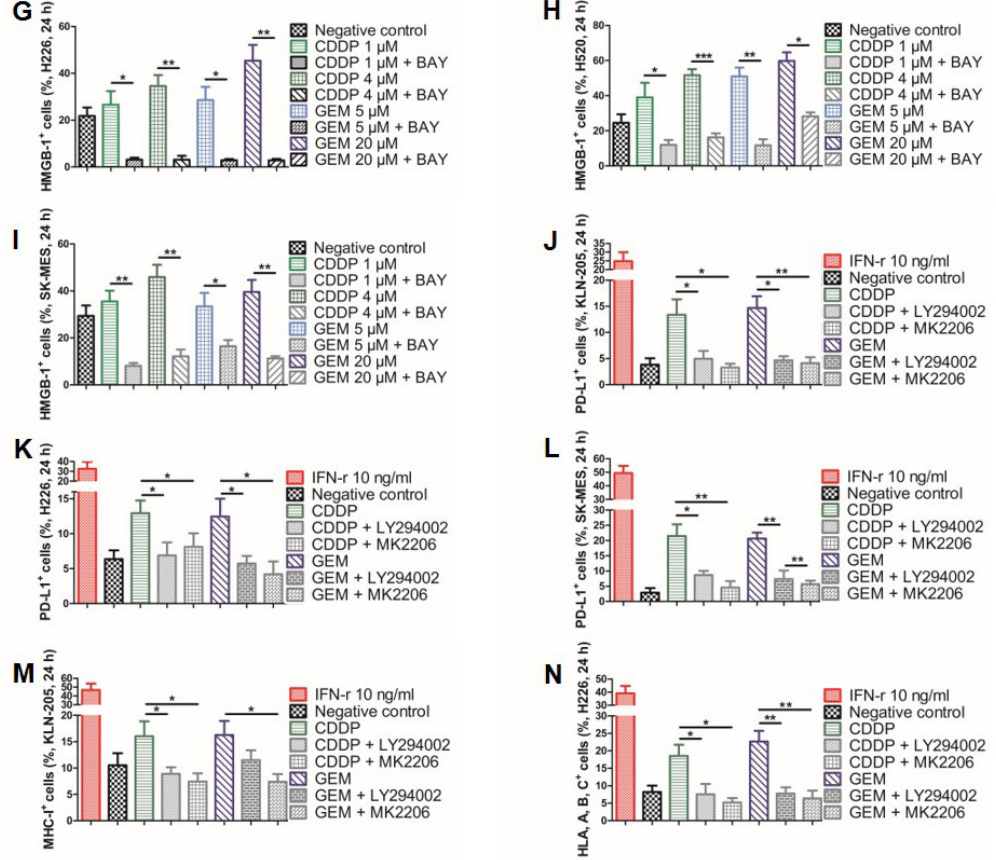

Figure 1 Low-dose chemotherapy may induce ICD via the PI3K/Akt/NF-KB signaling pathway in SQCLC cell lines. (A and B) RNA sequencing analysis to evaluate immune-related gene expression following low-dose chemotherapeutic treatment (CDDP $4 \mu \mathrm{M}$, GEM $20 \mu \mathrm{M}$, PTX $1 \mu \mathrm{M}$, DTX $1 \mu \mathrm{M}$ ) for 24 hours. (C-F) Percentage of SQCLC cells expressing cell surface PD$\mathrm{L} 1$, as revealed by FACS, after incubation with the indicated chemotherapy agents or controls (negative and positive) for 24 hours, with or without pretreatment with 1-3 $\mu$ M BAY 11-7082 (BAY), an NF- $\mathrm{BB}$ inhibitor, for 30 min-3 hours. (G-I) Percentage of (G) H226, (H) H520, and (I) SK-MES cells expressing cytoplasmic HMGB-1 by FACS, after incubation with the indicated chemotherapy agents or negative control for 24 hours, with or without concurrent treatment with $0.2 \mu M$ BAY. (J-L) Percentage of $(\mathrm{J}) \mathrm{KLN}-205$, (K) H226, and (L) SK-MES cells expressing cell surface PD-L1 by FACS, after incubation with the indicated

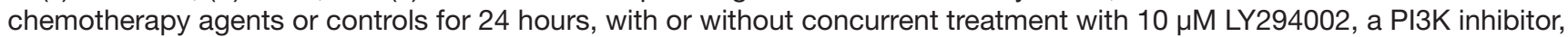
or $10 \mu \mathrm{M}$ MK-2206, an Akt inhibitor. (M) Percentage of KLN-205 cells expressing cell surface MHC-class I. (N) Percentage of $\mathrm{H} 226$ cells expressing cell surface HLA-A/B/C, after incubation with the indicated chemotherapy agents or controls for 24 hours, with or without concurrent treatment with $10 \mu \mathrm{M}$ LY294002 or $10 \mu \mathrm{M}$ MK-2206. Data are presented as the mean \pm SD; ${ }^{*} \mathrm{p}<0.05,{ }^{* *} \mathrm{p}<0.01,{ }^{* * *} \mathrm{p}<0.001$ for chemotherapy+inhibitor versus chemotherapy alone. CDDP, cisplatin; DTX, docetaxel; FACS, fluorescence-activated cell sorting; GEM, gemcitabine; HLA, human leukocyte antigen; HMGB-1, high mobility group box-1; ICD, immunogenic cell death; IFN, interferon; MHC, major histocompatibility complex; NF- $\mathrm{B}$, transcription factor nuclear factor kappa B; PD-L1, programmed death-ligand 1; PI3K, phosphatidylinositol 3-kinase; PTX, paclitaxel; SQCLC, squamous cell lung carcinoma. 
(4 $\mu \mathrm{M}$ CDDP, $20 \mu \mathrm{M}$ GEM, $1 \mu \mathrm{M}$ PTX, $1 \mu \mathrm{M}$ DTX) for 24 hours (figure $1 \mathrm{~B}$ and 178 genes were displayed in online supplemental table S1). Interestingly, we observed the differential expression of phosphatidylinositol 3-kinase (PI3K) /Akt and NF-kB (online supplemental table S1) after treatment. NF- $\mathrm{kB}$ is a pivotal regulator of both innate and adaptive immunity within the TIME. ${ }^{30-32}$ Thus, we further investigated whether the PI3K/Akt/ NF- $\kappa B$ signaling pathway was also involved in the immunomodulatory effect induced by low-dose chemotherapy. Consistent with this assumption, results from FACS data analysis revealed that the CDDP-induced or GEM-induced increases in PD-L1, MHC class I, HLA-A/B/C, and cytoplasmic HMGB-1 expression were abrogated in cells pretreated or co-incubated with BAY 11-7082 (NF-KB inhibitor), LY294002 (PI3K inhibitor), or MK2206 (Akt inhibitor), although to different extents (figure $1 \mathrm{C}-\mathrm{N}$ ). These observations suggested that treatment with lowdose chemotherapeutic agents induced ICD possibly via the PI3K/Akt/NF-KB signaling pathway, stimulating the release of more neoantigens and HMGB-1 from poorly immunogenic SQCLC cancer cells, in turn enhancing the antitumor immune response.

\section{Low-dose chemotherapy-induced secretion of HMGB-1 by SQCLC cells promotes maturation of dendritic cells}

Dendritic cells (DCs) are responsible for antigen presentation and play a central role in antitumor immunity. ${ }^{33}$ Damage-associated molecular patterns (DAMPs) are endogenous danger signals released by ICD cells. DAMPs exacerbate antitumor immune responses. Considering that HMGB-1 is one of the most well-described and important DAMPs, ${ }^{34}$ we assessed whether low-dose chemotherapeutic agents affected the maturation of murine BMDCs via HMGB-1. We incubated KLN-205 cells with low-dose chemotherapy agents for 30 hours and then dialyzed the conditioned culture media to remove the residual chemotherapeutic agent. Immature BMDCs were then incubated with the dialyzed media for another 24 hours. As a result, we observed an increased proportion of three mature BMDC subtypes, including

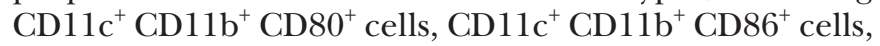
and $\mathrm{CD} 11 \mathrm{c}^{+} \mathrm{CD} 11 \mathrm{~b}^{+} \mathrm{MHC}$ class $\mathrm{II}^{+}$cells (figure $2 \mathrm{~A}$ and online supplemental figure S3), as well as total mature BMDCs (figure 2B). The data indicated that molecules secreted by cancer cells treated with low-dose chemotherapeutic drugs could induce DC maturation. Considering the critical role played by the DAMP HMGB-1 within the TIME, we next assessed HMGB-1 levels in the same conditioned media by ELISA. Compared with the negative control media, we observed a significant increase of HMGB-1 levels in the conditioned media from KLN-205 cells treated with low doses of any of the four chemotherapeutic drugs (figure 2C). Moreover, on stimulation of immature BMDCs with $50 \mathrm{ng} / \mathrm{mL}$ or $100 \mathrm{ng} /$ $\mathrm{mL}$ recombinant HMGB-1 for 24 hours, the percentages of BMDCs co-expressing the abovementioned markers also increased in a dose-dependent manner (figure 2D).
These data suggested that low-dose CDDP, GEM, DTX, and PTX could enhance DC maturation by inducing the release of HMGB-1 from SQCLC cells.

\section{Low-dose chemotherapy induces systemic antitumor immune responses and immunological memory}

Next, a syngeneic immunocompetent SQCLC mouse model was used to assess whether low-dose chemotherapy would have immune activation effects in vivo. We pretreated KLN-205-Luc cells with $1 \mu \mathrm{M}$ CDDP, 15 $\mu \mathrm{M}$ CDDP, or an equal volume of vehicle (PBS, negative control) for 72 hours. Pretreated cells were then injected into the right flanks of mice. Seven days later, splenocytes were harvested for an enzyme-linked immunospot (ELISPOT) assay to assess IFN- $\gamma$ secretion. Compared with the negative control and high-dose CDDP, splenocytes from mice injected with low-dose CDDP-pretreated KLN-205 cells secreted more IFN- $\gamma$ (figure 2E,F), indicative of an enhanced systemic antitumor immune response. At day 7, we rechallenged mice with untreated KLN-205-Luc cells in the opposite flank and monitored tumor growth dynamically by BLI in order to evaluate immunological memory resulting from CDDP pretreatment. Mice that had been injected with low-dose CDDPpretreated tumor cells exhibited less tumor burden (one out of seven mice) compared with control group mice (seven out of seven mice) and high-dose CDDP group mice (seven out of seven mice) (figure 2G). These data suggested that low-dose CDDP pretreatment was more immunogenic and protected mice against tumor recurrence. Thus, low-dose CDDP treatment could activate systemic immune responses and immunological memory in vivo.

\section{Low-dose monochemotherapy agents elicit an active SQCLC TIME in vivo}

Strategies, such as increasing PD-1/PD-L1 expression and reinforcing ELSs, which include $\mathrm{CD}^{+}$cytotoxic T cells, type I tumor-associated macrophages (TAMs), and DCs, are pivotal for rendering the TIME more sensitive to ICIs. Hence, we explored different dosages and timing strategies for the administration of chemotherapeutic agents in order to induce active antitumor immunity in syngeneic immunocompetent DBA-2J mice bearing SQCLC tumors, thereby providing an ICI response-supportive TIME. Currently, platinum-based doublet chemotherapy at the MTD combined with ICIs is the first-line care for patients with advanced SQCLC. To optimize this combination, a drug administration schedule was designed for the mouse study and is shown in online supplemental figure S4A. CDDP ( $5 \mathrm{mg} / \mathrm{kg}$, Q7D, 4 cycles) plus GEM $(160 \mathrm{mg} / \mathrm{kg}, \mathrm{Q} 4 \mathrm{D}, 4$ cycles) at the MTD significantly inhibited tumor growth (online supplemental figure S4B), but also resulted in a significantly increased number of type II TAMs, as detected by FACS (online supplemental figure S4C,D) and immunohistochemical analysis (online supplemental figure S4E), indicative of an immunosuppressive TIME that would be incompatible with 

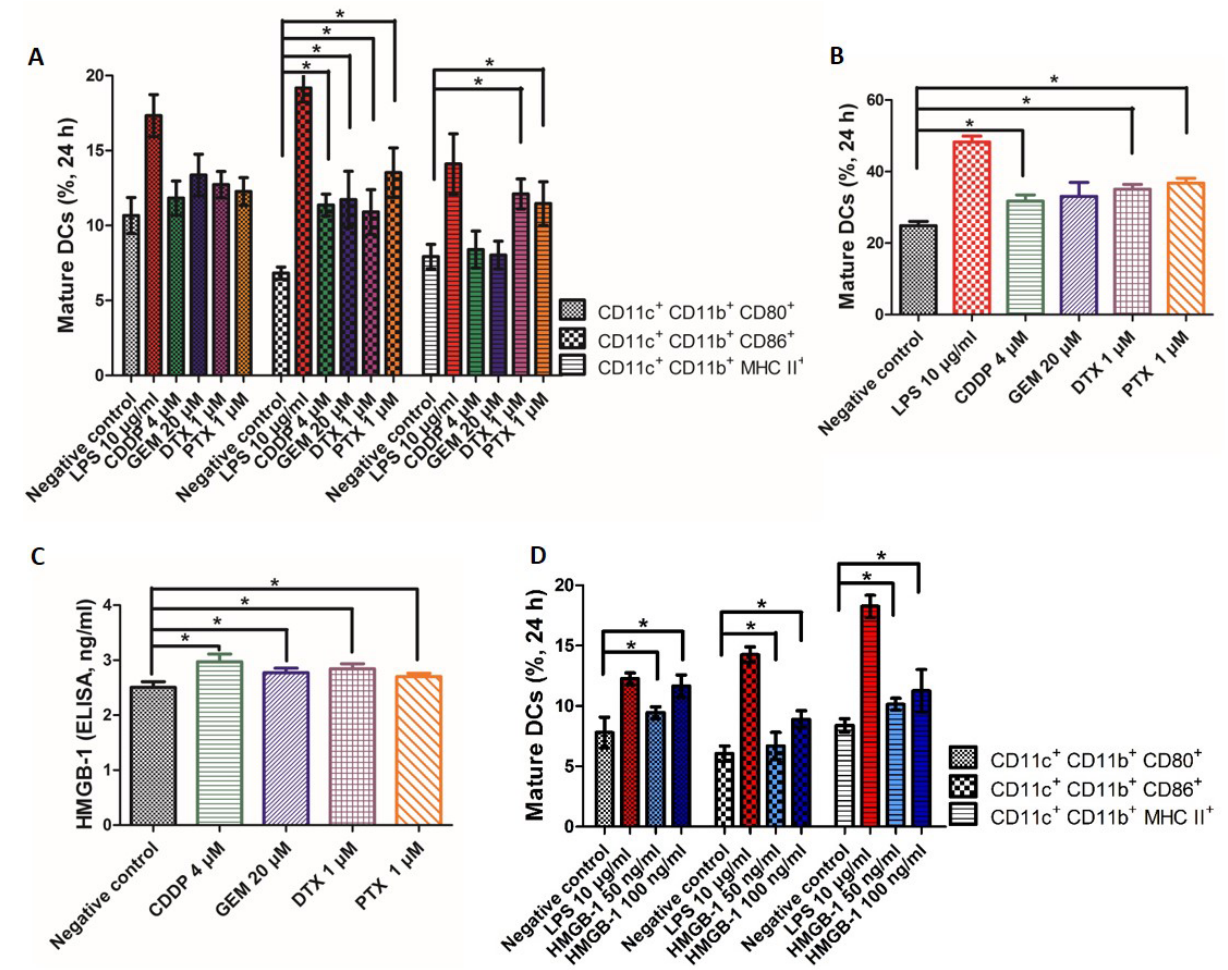

$\mathrm{E}$

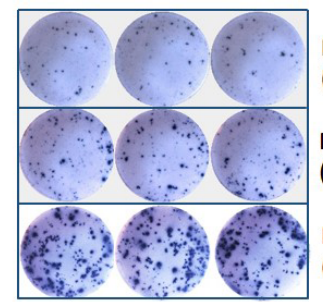

Negative control (PBS, 72 h)

Pretreated KLN-205 (15 $\mu \mathrm{M}$ CDDP, $72 \mathrm{~h})$

Pretreated KLN-205 (1 $\mu \mathrm{M}$ CDDP, $72 \mathrm{~h}$ )

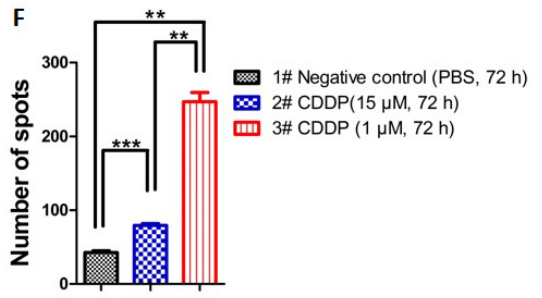

G

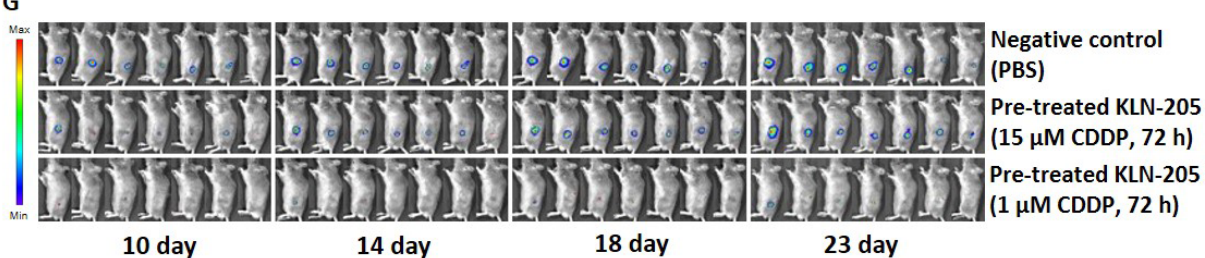

Figure 2 Low-dose chemotherapy induces HMGB-1 release by SQCLC cells, thereby promoting DC maturation (A-D) and inducing systemic immune responses as well as immunological memory in vivo (E,F). KLN 205-Luc cells were incubated with $4 \mu \mathrm{M}$ CDDP, $20 \mu \mathrm{M}$ GEM, $1 \mu \mathrm{M}$ DTX, or $1 \mu \mathrm{M}$ PTX for 30 hours, after which the conditioned media were dialyzed at $4^{\circ} \mathrm{C}$ for 24 hours using a 10,000-MWCO dialysis tubing to remove any residual chemotherapeutic drug. Immature murine bone marrowderived dendritic cells (BMDCs) cultured in 6 -well plates $\left(1 \times 10^{6} \mathrm{cell} / \mathrm{sL}\right.$ ) were then cultured in unconditioned media (negative control), media containing LPS (10 $\mathrm{\mu g} / \mathrm{mL}$, positive control), or different conditioned media, and were harvested 24 hours later. The maturation state of BMDCs was evaluated by measuring the cell surface co-expression of $C D 11 \mathrm{c}^{+} \mathrm{CD} 11 \mathrm{~b}^{+} \mathrm{CD} 80^{+}, \mathrm{CD}_{11 \mathrm{c}^{+}}$

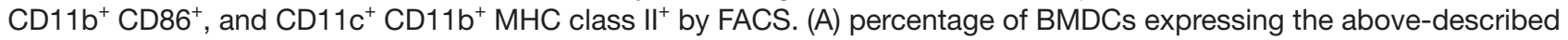
combinations of three different cell maturation biomarkers after the different stimuli, as revealed by FACS. (B) Proportion of total mature BMDCs in each group after the different stimuli. (C) HMGB-1 levels in the media after treatment with different chemotherapeutic agents for 30 hours, as assessed by ELISA. (D) Immature BMDCs were stimulated with $50 \mathrm{ng} / \mathrm{mL}$ or $100 \mathrm{ng} /$

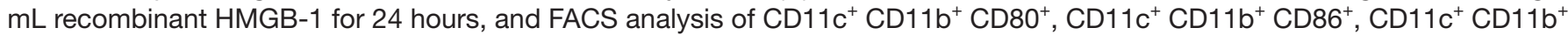
MHC class II $^{+}$expression was carried out. (E,F) Murine KLN-205-Luc cells were pretreated with $1 \mu \mathrm{M}$ CDDP, $15 \mu \mathrm{M}$ CDDP for 72 hours or with PBS as the negative control. These pretreated cells $\left(1 \times 10^{6}\right)$ were subcutaneously injected into the right flanks of mice. Splenocytes were harvested after 7 days, and IFN- $\gamma$ secretion was evaluated using ELISPOT, according to the kit manufacturer's protocol. (G) The aforementioned mice were rechallenged with $2 \times 10^{5}$ live KLN-205 cells in the opposite flank, and tumor growth was monitored using vernier calipers and BLI. Data are presented as mean \pm SD. No significance, ${ }^{*} p<0.05$; ${ }^{* *} p<0.01 ;{ }^{* *} p<0.001$. BLI, bioluminescence imaging; CDDP, cisplatin; DCs, dendritic cells; DTX, docetaxel; ELISPOT, enzymelinked immunospot; FACS, fluorescence-activated cell sorting; GEM, gemcitabine; HMGB-1, high mobility group box-1; IFN- $\gamma$, interferon-gamma; LPS, lipopolysaccharide; Luc, luciferase; MHC, major histocompatibility complex; PBS, phosphate-buffered saline; PTX, paclitaxel. 
subsequent or concurrent immunotherapy. Next, based on the recommended dosages of the National Comprehensive Cancer Network guidelines and a human-mouse dosage conversion formula, ${ }^{12}{ }^{35}$ we derived adapted MTD dosages for application in mice. We then consulted previously published data to further define whether mice can withstand these dosages. Afterwards, we tested the dosages in mice to confirm that a mean weight loss of less than $10 \%$ of the initial body weight would be observed after intraperitoneal administration. The low dose was approximately one-third of the high dose, as shown in online supplemental table S2. Next, we attempted to compare the specific differences of the TIME after low-dose CDDP $2.8 \mathrm{mg} / \mathrm{kg}$, high-dose CDDP $8.4 \mathrm{mg} / \mathrm{kg}$, low-dose GEM $60 \mathrm{mg} / \mathrm{kg}$, high-dose GEM $240 \mathrm{mg} / \mathrm{kg}$, low-dose PTX 11 $\mathrm{mg} / \mathrm{kg}$, high-dose PTX $33 \mathrm{mg} / \mathrm{kg}$, low-dose DTX $11 \mathrm{mg} /$ $\mathrm{kg}$, high-dose DTX $33 \mathrm{mg} / \mathrm{kg}$ administration, or vehicle. As shown in figure $3 \mathrm{~A}, \mathrm{~B}$ and online supplemental figure S5, we observed that low-dose CDDP, GEM, PTX, DTX contributed to an enhanced intratumoral $\mathrm{CD}_{4} 5^{+} \mathrm{CD}^{+}$ and $\mathrm{CD} 45^{+} \mathrm{CD}^{+} \mathrm{CD}^{+}$cell infiltration (cytotoxic $\mathrm{T}$ cells) after 30 hours, especially PTX and DTX. In contrast, highdose treatment promoted intratumoral $\mathrm{CD} 45^{+} \mathrm{CD} 3^{+} \mathrm{CD} 4^{+}$ $\mathrm{CD}^{2} 5^{+}$regulatory $\mathrm{T}$ cell (Treg) infiltration, indicative of enhanced immunosuppression within the tumor microenvironment. In addition, as illustrated in figure 3C,D, patients with treatment-naive SQCLC received either MTD albumin-PTX (at day 0)+nivolumab (routine administration) or low-dose albumin-PTX (1/2 MTD at day 0 and day 7)+nivolumab (routine administration). Peripheral blood mononuclear cells (PBMCs) were isolated from patient blood by density gradient centrifugation according to a standard procedure, ${ }^{36}$ and were subjected to FACS analysis. Before treatment (day 0), baseline PBMCs were evaluated. At day 7 (before the second lowdose albumin-PTX administration), PBMCs were once again detected. We observed an upregulation of cytotoxic T cells after low-dose albumin-PTX+nivolumab treatment, which was consistent with the data obtained from animal models. Thereafter, we assessed the potential of low-dose chemotherapeutic treatment to elicit robust ELS activation responses and enhance subsequent immunotherapy. Twenty-four hours after one low-dose injection, CDDP (2.8 $\mathrm{mg} / \mathrm{kg}$ ), GEM (80 mg/kg), DTX (11 mg/kg), or PTX (11 $\mathrm{mg} / \mathrm{kg}$ ) monotherapies exerted distinct immunomodulatory effects in vivo, enhancing the percentages of $\mathrm{CD} 45^{+}$ PD- $1^{+}$cells, CD11 ${ }^{+} \mathrm{CD} 3^{+}$cells (activated DCs), CD11 ${ }^{+}$ MHC-class $\mathrm{II}^{+}$cells (activated DCs), $\mathrm{CD} 11 \mathrm{~b}^{+} \mathrm{F} 4 / 80^{+} \mathrm{CD}_{11 \mathrm{c}^{+}}$ cells (type I TAMs), $\mathrm{CD}^{+} \mathrm{CD}^{+} \mathrm{T}$ cells, and $\mathrm{CD} 3^{+} \mathrm{CD} 4^{+} \mathrm{T}$ cells in both tumor and spleen tissue (figure 4 ). The above data provided evidence for the potential of upfront lowdose monochemotherapeutic agent treatment in combination with subsequent immunotherapy for the treatment of SQCLC. Moreover, our data suggested that 24 hours after upfront low-dose monochemotherapy may be good timing for initiating immunotherapy.
Sequential low-dose CDDP exerts more pronounced synergistic antitumor effects than MTD CDDP, when combined with anti-PD-1 mAb in vivo

Since the aforementioned low-dose monotherapies elicited robust antitumor immune responses and induced greater PD-1/PD-L1 expression in vivo, the following treatment schedule was designed, as illustrated in figure 5A. We observed that the sequential administration of upfront low-dose CDDP and anti-PD-1 ICIs resulted in more pronounced synergistic antitumor responses compared with MTD CDDP plus anti-PD-1 mAb (figure 5B-D and F). Moreover, the weight loss observed following this drug combination was lesser (figure 5E), and treatment did not significantly elevate serum alanine transaminase or aspartate aminotransferase, nor did it negatively affect RBCs, hemoglobin, and platelets in whole blood (figure $5 \mathrm{M}-\mathrm{Q}$ ). As shown in figure 5G-L and online supplemental figure $\mathrm{S} 6$, after three treatment cycles with CDDP/anti-PD-1 mAb combination chemoimmunotherapy, we observed increased numbers of CD $45^{+}$ $\mathrm{CD}^{+}$cells (lymphocytes), $\mathrm{CD} 45^{+} \mathrm{CD}^{+} \mathrm{CD}^{+}$cells (cytotoxic $\mathrm{T}$ cells), $\mathrm{CD} 45^{+} \mathrm{CD} 3^{+} \mathrm{CD} 8^{+} \mathrm{PD}-1^{+}$cells (targeted by anti-PD-1/PD-L1 mAbs), CD11 $\mathrm{c}^{+} \mathrm{CD} 3^{+}$cells, and CD11 $\mathrm{c}^{+}$ $\mathrm{CD}^{+} 6^{+}$(activated DCs), indicative of enhanced antitumor immune responses and upregulated therapeutic target expression when compared with mice that received MTD CDDP combined with anti-PD-1 mAb.

\section{Upfront metronomic DTX plus anti-PD-1 mAb inhibits SQCLC growth more effectively than the MTD regimen combined with anti-PD-1 mAb}

Currently, MTD chemotherapy combined with ICIs is the standard treatment for patients with SQCLC. Hence, we explored whether upfront metronomic (low-dose and frequent administration) chemotherapy would outperform routine MTD regimens when both were combined with anti-PD-1 mAb treatment. Considering that lowdose DTX elicited relatively stronger antitumor immune responses in vivo than the other agents tested (figures 3 and 4), we selected DTX for further study. The treatment schedule we used is illustrated in figure 6A. Using the human-mouse dosage conversion formula referred to above, a dosage of $33 \mathrm{mg} / \mathrm{kg}$ DTX for each mouse was the equivalent of the routine standard dose (ie, MTD) of DTX in human adults. Among the six treatment groups, sequential administration of metronomic DTX followed by anti-PD-1 mAb exhibited the best therapeutic effects (figure 6C-F). Moreover, metronomic DTX induced lower body weight loss compared with MTD DTX, when both were combined with ICI (figure 6B). We further digested tumors to create single-cell suspensions and evaluated immune-related markers by FACS analysis. Intratumoral TAMs (figure 6G), type I TAMs (figure 6H), and activated DCs (figure 6J-L) were all significantly elevated in mice treated with metronomic DTX plus anti-PD-1 mAb. Elevated levels of type II macrophages were observed in tumors (figure 6I) of mice treated with MTD DTX, suggestive of significant immunosuppression. Additionally, in 
A Day 0, 1\# Negative control (equivalent solvent), 2\# high dose CDDP $8.4 \mathrm{mg} / \mathrm{kg}$, 3\# low dose CDDP $2.8 \mathrm{mg} / \mathrm{kg}$, 4\# high dose GEM $240 \mathrm{mg} / \mathrm{kg}$, 5\# low dose GEM $80 \mathrm{mg} / \mathrm{kg}$, 6\# high dose PTX $33 \mathrm{mg} / \mathrm{kg}$, 7\# low dose PTX 11 mg/kg, 8\# high dose DTX 33 mg/kg, 9\# low dose DTX 11 mg/kg,

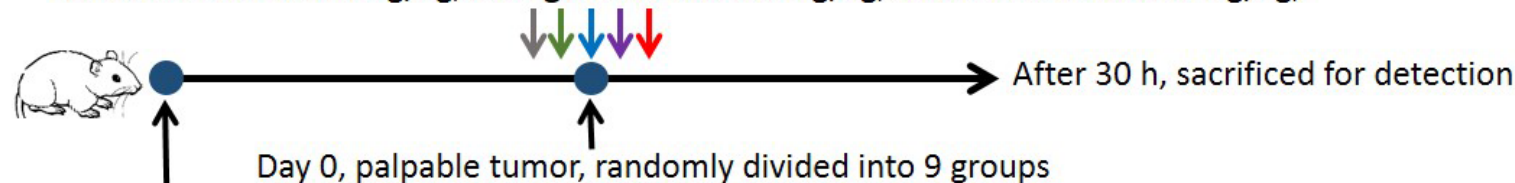

Day $-18,5 \times 10^{5} \mathrm{KLN}-205-$ Luc inoculation

B

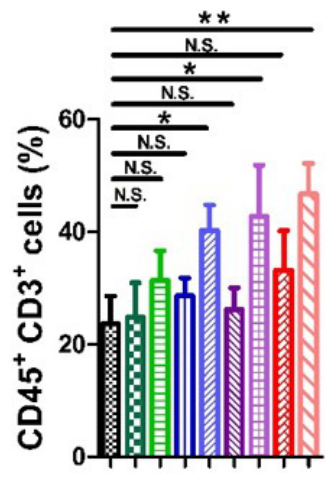

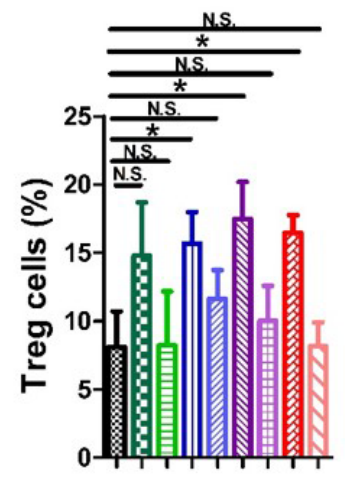

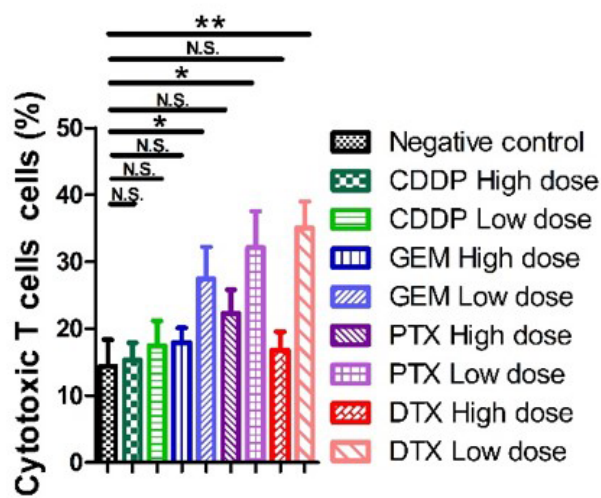

C

Day 0, before treatment, baseline PBMCs was

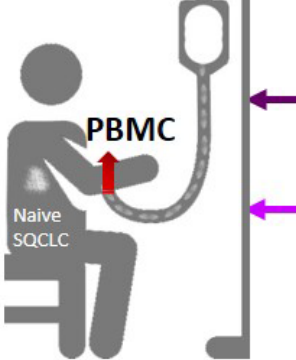
detected by FACS.

MTD albumin-PTX + nivolumab (routine) OR Low dose albumin-PTX + nivolumab (routine) (1/2 MTD at day 0 and day 7$)$

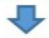

Day 7, before 2th low dose albumin-PTX, PBMCs was again detected by FACS

Day 0 and day 7 , Comparison

Indicator:

Cytotoxic T cells (\%)

Treg cells (\%)
D MTD albumin-PTX + nivolumab Low albumin-PTX + nivolumab
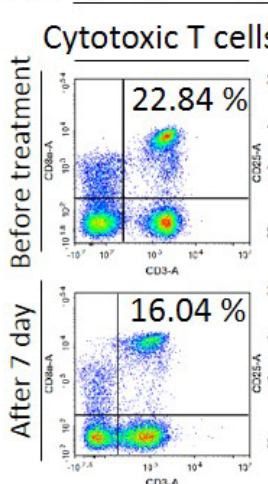
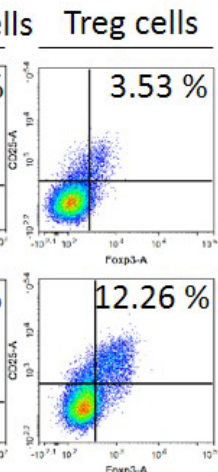
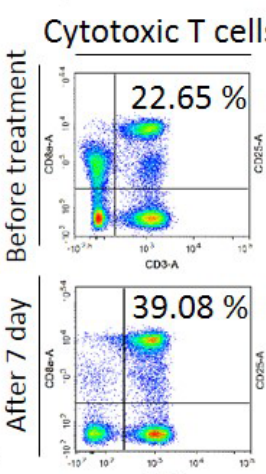

s Treg cells
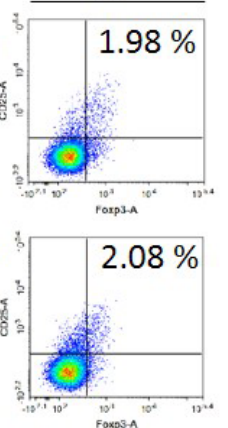

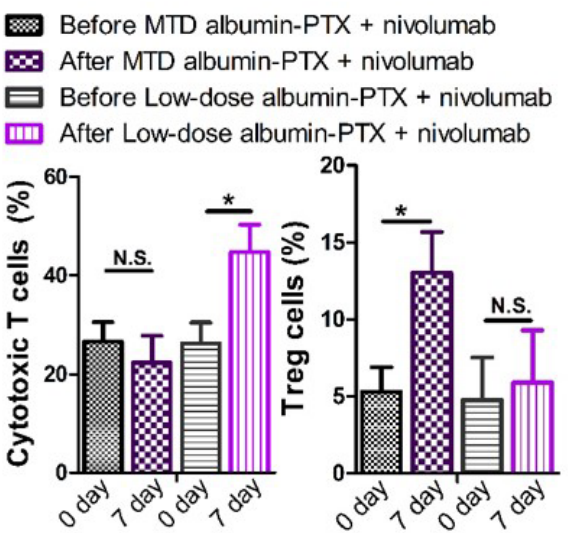

Figure 3 High-dose SQCLC monochemotherapy induce greater immunosuppression compared with low-dose regimens in vivo. (A) Chemotherapy treatment scheme. Syngeneic mouse models were established as mentioned above. When tumors became palpable, mice were randomly divided into nine groups receiving an intraperitoneal injection of low-dose CDDP 2.8 mg/kg, high-dose CDDP 8.4 mg/kg, low-dose GEM 60 mg/kg, high-dose GEM 240 mg/kg, low-dose PTX 11 mg/kg, highdose PTX $33 \mathrm{mg} / \mathrm{kg}$, low-dose DTX $11 \mathrm{mg} / \mathrm{kg}$, high-dose DTX $33 \mathrm{mg} / \mathrm{kg}$, or vehicle. (B) After 30 hours, tumors were harvested for FACS analysis. (C,D) Patients with reatment-naïve SQCLC received either MTD albumin-PTX (at day 0)+nivolumab (routine administration) or low-dose albumin-PTX (1/2 MTD at day 0 and day 7)+nivolumab (routine administration). Before treatment (day 0), baseline PBMCs were evaluated by FACS. At day 7 (before second low-dose albumin-PTX), PBMCs were again assessed by FACS. Cell fractions (cytotoxic T cells and Treg cells) before and after treatment were then compared. Data are presented as mean \pm SD. N.S., no significance, ${ }^{*} p<0.05 ;{ }^{* \star} p<0.01 ;{ }^{* \star *} p<0.001$. CDDP, cisplatin; DTX, docetaxel; FACS, fluorescence-activated cell sorting; GEM, gemcitabine; Luc, luciferase; MTD, maximum tolerated dose; PBMC, peripheral blood mononuclear cell; PTX, paclitaxel; SQCLC, squamous cell lung carcinoma; Treg, regulatory T cell. 
A Day 0, 1\# Negative control (equivalent solvent), 2\# CDDP $2.8 \mathrm{mg} / \mathrm{kg}$,

3\# GEM 80 mg/kg, 4\# DTX 11 mg/kg, 5\# PTX 11 mg/kg

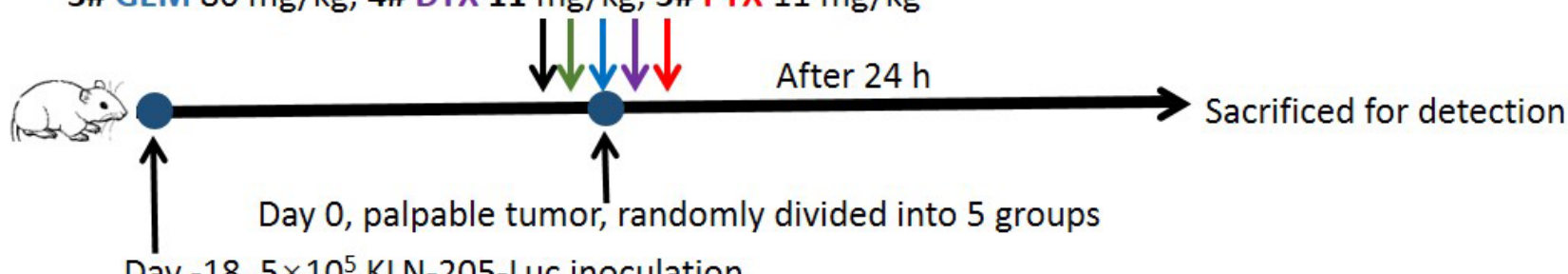

Day $-18,5 \times 10^{5} \mathrm{KLN}-205$-Luc inoculation
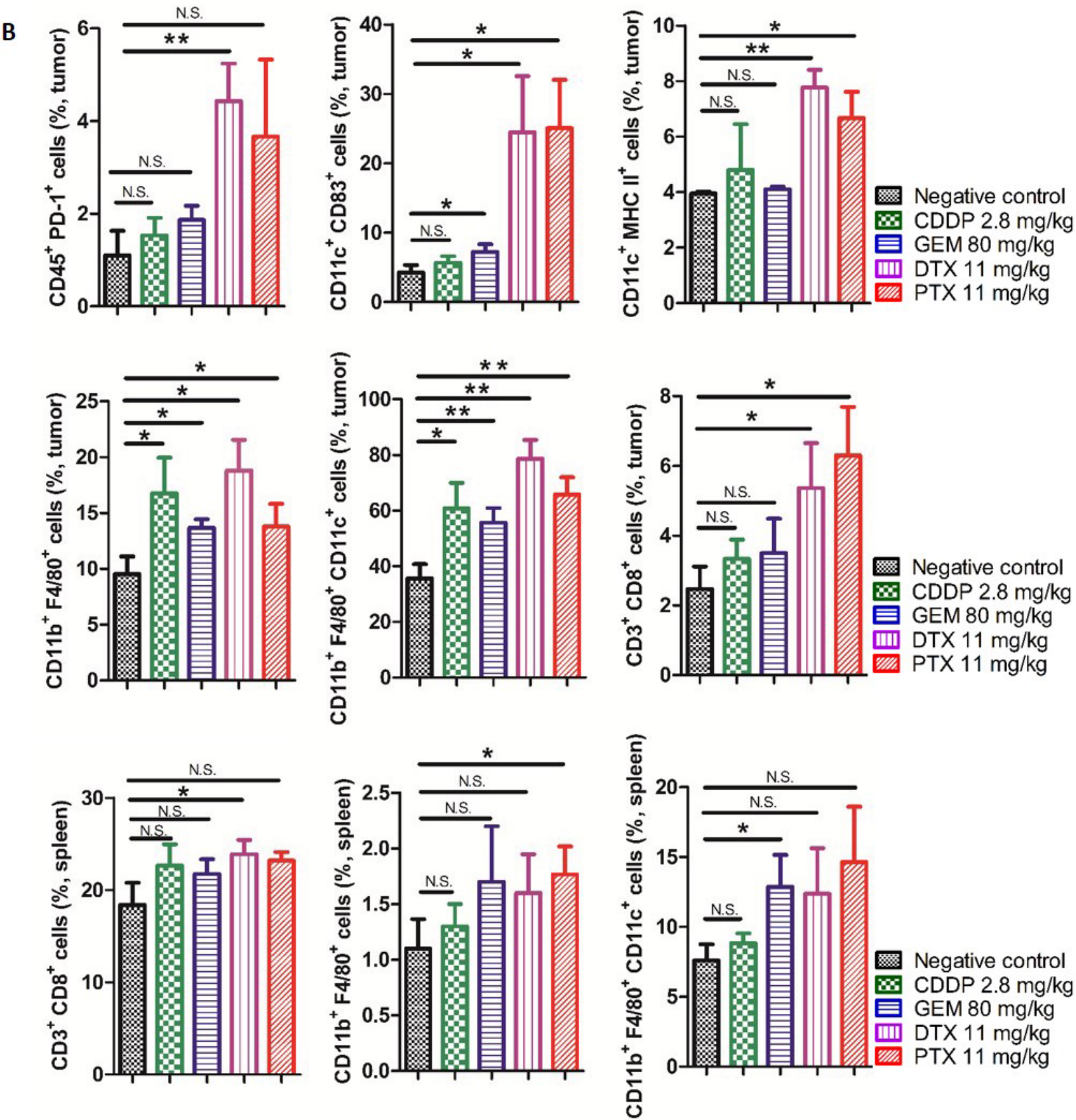

Figure 4 Low-dose SQCLC monochemotherapy elicits an active TIME in vivo. (A) Chemotherapy treatment scheme. The syngeneic mouse models were established as previously described. When the tumors were palpable, mice were randomly divided into five groups and then received an intraperitoneal injection of CDDP $2.8 \mathrm{mg} / \mathrm{kg}$, GEM $80 \mathrm{mg} / \mathrm{kg}$, DTX $11 \mathrm{mg} / \mathrm{kg}$, PTX $11 \mathrm{mg} / \mathrm{kg}$, or vehicle. (B) After 24 hours, the tumor and spleen tissues were harvested for FACS analysis. Data are presented as mean \pm SD. N.S., no significance, ${ }^{*} p<0.05 ;{ }^{* *} p<0.01 ;{ }^{* * *} p<0.001$ versus negative control. CDDP, cisplatin; DTX, docetaxel; FACS, fluorescence-activated cell sorting; GEM, gemcitabine; Luc, luciferase; PTX, paclitaxel; SQCLC, squamous cell lung carcinoma; TIME, tumor-immune microenvironment. 
A Day $-10,5 \times 10^{5} \mathrm{KLN}-205-$ Luc inoculation
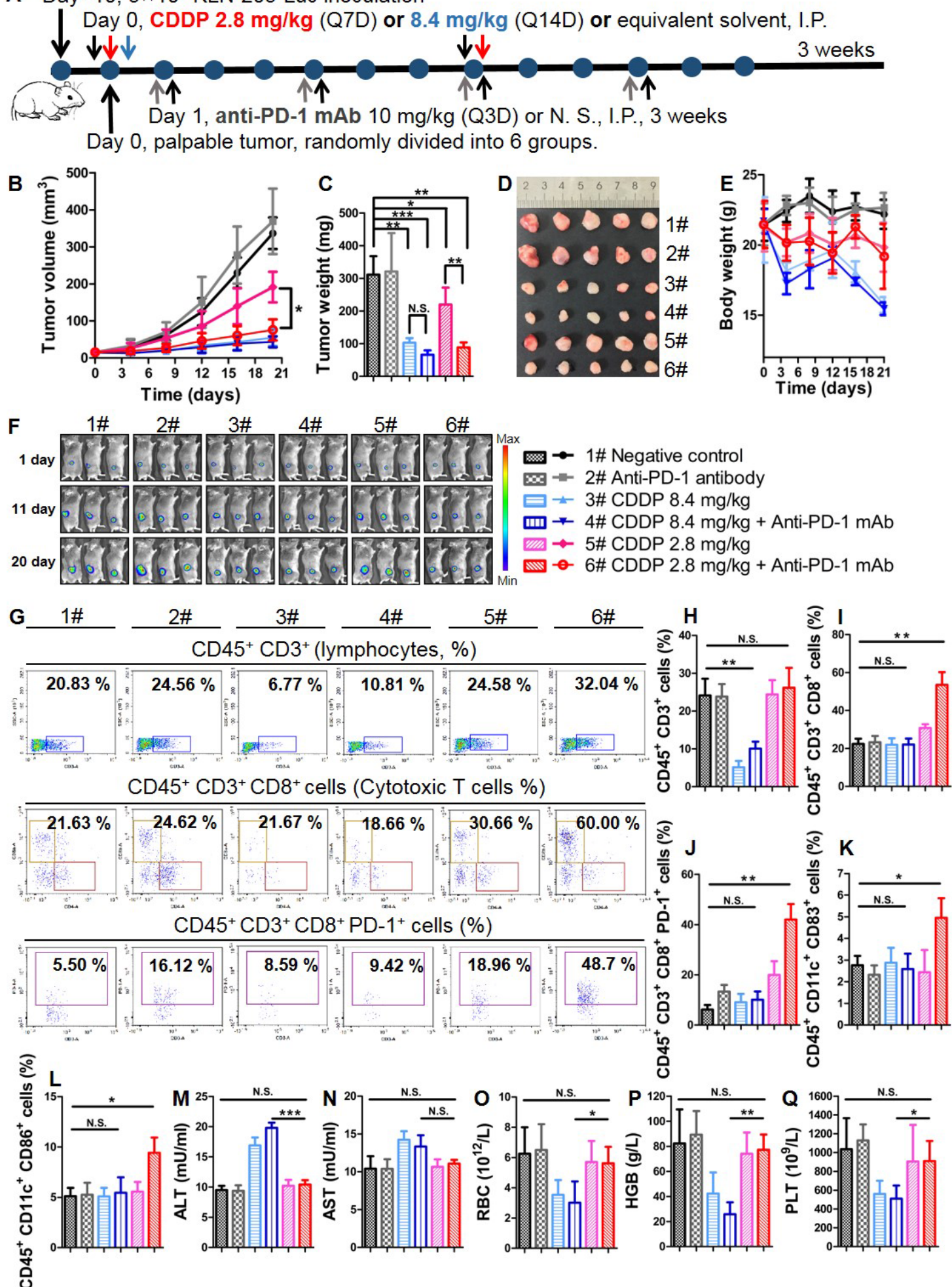

Figure 5 Combined with anti-PD-1 mAb, sequential low-dose CDDP exerts greater synergistic antitumor effects than MTD CDDP. (A) Treatment scheme. Upfront low-dose CDDP (2.8 mg/kg, Q7D), MTD CDDP (8.4 mg/kg, Q14D), low-dose or MTD CDDP combined with an anti-PD-1 mAb, anti-PD-1 mAb alone, and vehicle control were injected intraperitoneally (I.P.). (B) Tumor growth curves during treatment. (C,D) Tumor weight and specimens after treatment. (E) Body weight changes during treatment. (F) Tumor growth monitored by BLI. (G-L) Single-cell tumor suspensions were used for FACS to evaluate intratumoral CD45 ${ }^{+} \mathrm{CD}^{+}$cells, CD $45^{+} \mathrm{CD}^{+} \mathrm{CD} 8^{+}$cells, $\mathrm{CD} 45^{+} \mathrm{CD}^{+} \mathrm{CD} 8^{+} \mathrm{PD}-1^{+}$cells, $\mathrm{CD} 11 \mathrm{c}^{+} \mathrm{CD} 83^{+}$cells, and $\mathrm{CD} 11 \mathrm{c}^{+} \mathrm{CD} 6^{+}$cells. (M-Q) ALT and AST in serum, RBCs, HGB, and PLT in whole blood after treatment. Data are presented as mean \pm SD. N.S., no significance, ${ }^{*} \mathrm{p}<0.05 ;{ }^{* *} \mathrm{p}<0.01 ;{ }^{* * *} \mathrm{p}<0.001$. ALT, alanine transaminase; AST, aspartate aminotransferase; BLI, bioluminescence imaging; CDDP, cisplatin; FACS, fluorescence-activated cell sorting; HGB, hemoglobin; mAb, monoclonal antibody; MTD, maximum tolerated dose; PD-1, programmed death 1; PLT, platelet; RBC, red blood cell. 
A Day $-16,5 \times 10^{5} \mathrm{KLN}-205-\mathrm{Luc}$ inoculation

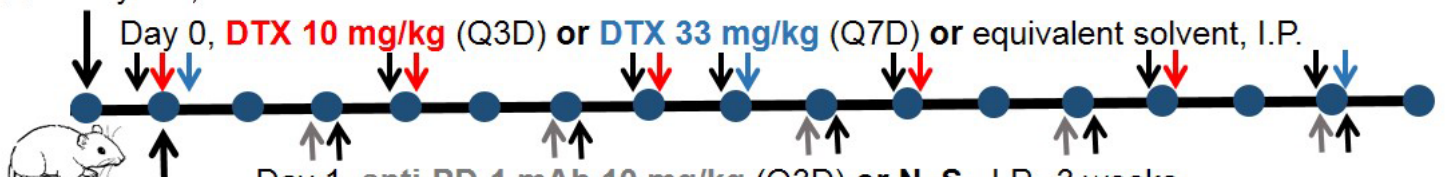

Day 1, anti-PD-1 mAb 10 mg/kg (Q3D) or N. S., I.P., 3 weeks

Day 0 , palpable tumor, randomly divided into 6 groups.
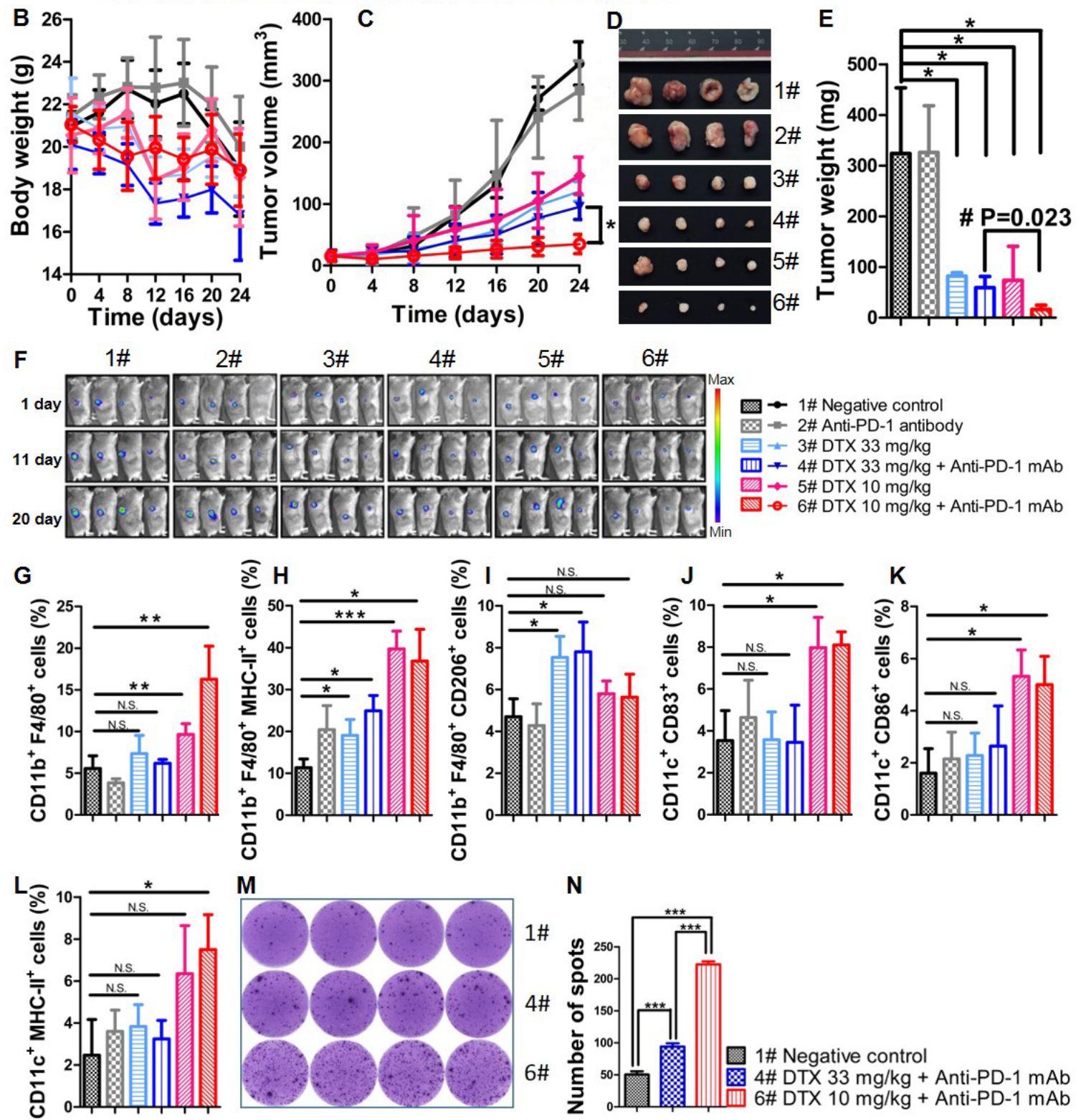

Figure 6 Combined with anti-PD-1 mAb, upfront metronomic DTX exerts greater tumor inhibitory effects than routine MTD DTX. (A) Treatment scheme. Upfront low-dose metronomic DTX (10 mg/kg, Q4D), routine MTD DTX (33 mg/kg, Q7D), equivalent to the standard dose administered to patients, low-dose or MTD DTX combined with an anti-PD-1 mAb, anti-PD-1 mAb alone, or vehicle control were injected intraperitoneally (I.P.) in mice. (B) Body weight changes during treatment. (C) Tumor growth curves during treatment. (D,E) Tumor specimens and weight after treatment. (F) Tumor growth monitored by BLI. (G-L) After treatment, single-cell tumor suspensions were used for FACS. (G) CD11 $b^{+} F 4 / 80^{+}$cells (TAMs). (H) CD11 b $\mathrm{b}^{+} \mathrm{F} 4 / 80^{+} \mathrm{MHC}$ - II+cells

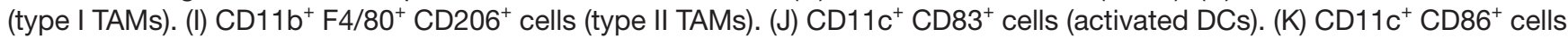
(activated DCs). (L) CD11 $\mathrm{c}^{+}$MHC-II cells (activated DCs). (M,N) Mice harboring established OVA-expressing KLN-205 tumors were treated with low-dose+anti-PD-1 mAb, high-dose DTX+anti-PD-1 mAb, or vehicle control by I.P. injection. After 3-week treatment, homolateral tumor-draining lymph nodes were prepared into single-cell suspensions, and subjected to ELISPOT assays. Data are presented as mean \pm SD. N.S., no significance, ${ }^{*} p<0.05 ;{ }^{* \star} p<0.01 ;{ }^{\star \star \star} p<0.001$. BLI, bioluminescence imaging; DCs, dendritic cells; DTX, docetaxel; ELISPOT, enzyme-linked immunospot; FACS, fluorescence-activated cell sorting; Luc, luciferase; mAb, monoclonal antibody; MHC, major histocompatibility complex; MTD, maximum tolerated dose; PD-1, programmed death 1; TAMs, type I tumor-associated macrophages. 
order to evaluate tumor antigen-specific $\mathrm{T}$ cell responses, mice harboring established OVA-expressing KLN205 tumors were treated with low-dose DTX+anti-PD-1 mAb, high-dose DTX+anti-PD-1 mAb, or vehicle control by intraperitoneal injection as mentioned above. After 3-week treatment, homolateral tumor-draining lymph nodes were prepared into single-cell suspensions and subjected to ELISPOT assays for detecting IFN- $\gamma$ secretion. As shown in figure $6 \mathrm{M}, \mathrm{N}$, significantly higher numbers of spots were observed after low-dose DTX+anti-PD-1 mAb treatment, compared with other two groups. The above data indicated that metronomic DTX was more effective in triggering immune activation, and its antitumor effects were enhanced to a greater extent when combined with anti-PD-1 mAb as compared with MTD DTX plus antiPD-1 mAb.

\section{Macrophages, $\mathrm{CD}^{+} \mathrm{T}$ cells, and gut microbiota are involved in the synergistic antitumor effects of metronomic DTX and anti-PD-1 mAb}

To further elucidate the potential mechanisms underlying the synergy of upfront metronomic DTX and antiPD-1 mAb in vivo, depletion of macrophages and $\mathrm{CD} 8^{+} \mathrm{T}$ cells was performed before chemoimmunotherapy. The detailed dosing schedule is described in figure 7A. As shown in figure 7B,C, the tumor growth delay on metronomic DTX combined with anti-PD-1 mAb was significantly altered by depletion pretreatment, which indicated that both macrophages and $\mathrm{CD} 8^{+} \mathrm{T}$ cells may play pivotal roles in mediating the synergistic antitumor effects. In addition, we investigated whether the anti-PD-1 mAb and anti-PD-L1 mAb treatment would lead to different outcomes when combined with metronomic DTX. As illustrated in figure 7B-E, both combination therapies induced antitumor activity, and the difference between them was not significant $(p>0.05)$. We then collected mouse feces at the end of treatment and carried out next-generation RNA sequencing (16S rRNA hypervariable regions). The reduction in intestinal gut microbiota diversity observed after MTD DTX plus anti-PD-1 mAb seemed greater (figure $7 \mathrm{~F}, \mathrm{p}=0.11$ ) compared with that following metronomic DTX in combination with antiPD-1 mAb. However, the difference was not significant. In addition, through principal component analysis, we observed an abnormal microbial composition in samples from the MTD DTX and anti-PD-1 mAb combination group (figure 7G). The above data suggested that the gut microbiota may also be involved in the observed synergistic antitumor effects.

\section{DISCUSSION}

To date, no studies on the optimization of chemoimmunotherapy strategies for SQCLC have been reported. In this work, we mainly focused on exploring how to turn 'cold' SQCLCs into ICI-sensitive tumors, thus enhancing the efficacy of chemoimmunotherapy through an improved synergy between chemotherapy and ICIs. Further, we aimed to identify possible underlying functional mechanisms both in vitro and in vivo. This is the first study to demonstrate that the upfront metronomic chemotherapy can synergize with immunotherapy in order to achieve substantially better therapeutic effects in SQCLC. This was principally achieved through the recruitment and activation of $\mathrm{CD}^{+} \mathrm{T}$ cells and DCs, polarization of type I macrophages, and the maintenance of intestinal gut microbiota diversity and normal composition.

Reaching the balance between immune activation and tumor inhibition is of great importance for optimizing chemoimmunotherapy. Herein, we explored ways through which chemotherapy might elicit robust antitumor immune responses in syngeneic SQCLC mouse models. At low dosages, all chemotherapeutics tested exerted robust in vivo ELS modulation in spleen and tumor tissue. For chemotherapy to induce an optimal TIME, the order of drug administration is critical. However, little information is available for the prediction of combination treatment efficacy and associated effects. We found that the administration of low-dose chemotherapy 24 hours prior to immunotherapy might be a good regimen for chemoimmunotherapy, as it not only decreased drug-associated toxicity during concurrent administration but also provided a sufficient time window for low-dose chemotherapy to activate the TIME.

We further compared the differences between the immunostimulatory effects of routine MTD and low-dose DTX regimens. The data revealed that low-dose DTX could significantly activate the TIME and induce higher expression of anti-PD-1/PD-L1 mAb therapeutic targets. Conversely, immunosuppressive effects were observed with routine MTD DTX. When combined with anti-PD-1 $\mathrm{mAb}$ in a sequential manner, low-dose metronomic DTX was more effective and less toxic compared with MTD DTX. With regard to the efficacy and tolerability of different DTX regimens, previous publications reported that low-dose and dense DTX $\left(33.3 \mathrm{mg} / \mathrm{m}^{2}\right.$, weekly) led to significantly lower lymphocytopenia in patients with NSCLC when compared with MTD DTX $\left(75 \mathrm{mg} / \mathrm{m}^{2}\right.$, every 3 weeks), ${ }^{37}$ and similar results were reported in a meta-analysis. ${ }^{38}$ Taken together, these results suggest that metronomic chemotherapy may be more favorable for subsequent immunotherapy compared with routine MTD chemotherapy.

Based on the above-described data, we suggest that the sequential combination of low-dose metronomic DTX and anti-PD-1 mAb may be a promising therapeutic alternative for patients with SQCLC. Considering the differences between humans and mice models, we assessed PBMCs obtained from patients with SQCLC before and after MTD albumin-PTX (at day 0)+nivolumab (routine administration) or low-dose albumin-PTX (1/2 MTD at day 0 and day 7)+nivolumab (routine administration). The data revealed an upregulation of cytotoxic T cells after low-dose albumin-PTX+nivolumab treatment, while MTD albumin-PTX+nivolumab contributed to more severe immunosuppression (increased Treg cell infiltration), 
A

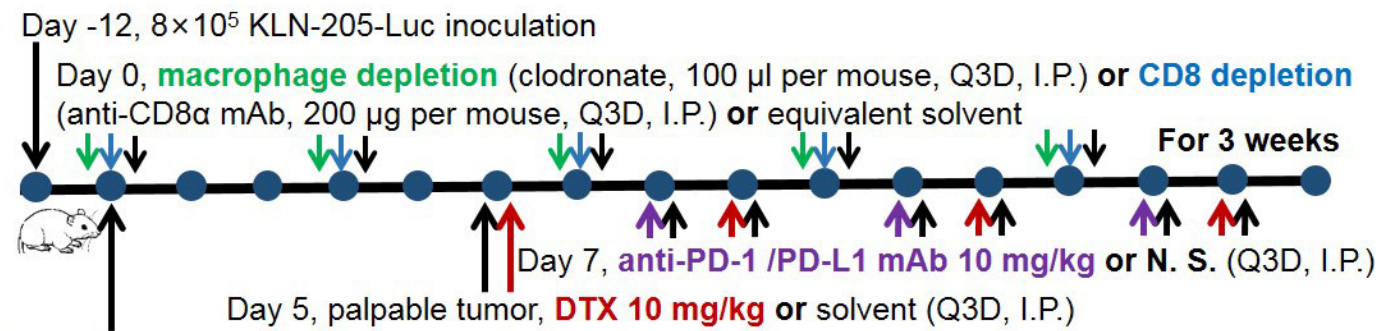

Day 0, palpable tumor, randomly divided into 6 groups.
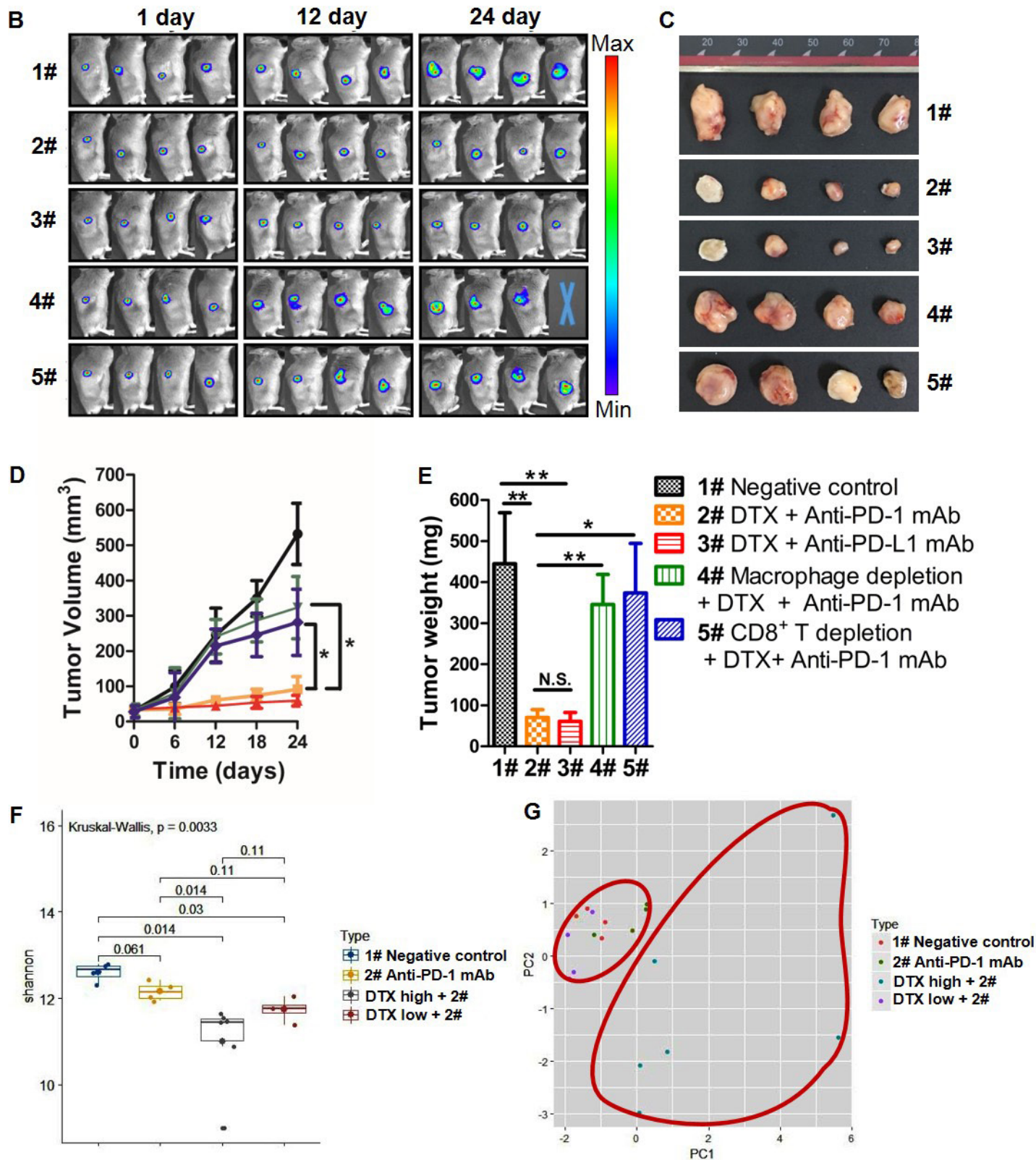

Figure 7 Macrophages, CD8 ${ }^{+} \mathrm{T}$ cells, and gut microbiota may contribute to the synergistic antitumor effects of DTX plus anti-PD-1 mAb. (A) Schematic of dosing schedule. Macrophage depletion (clodronate, neutral clodronate liposomes, $100 \mathrm{\mu L}$ per mouse, Q3D, intraperitonially (I.P.)) and CD8 ${ }^{+}$T cell depletion (in vivo anti-CD $8 \alpha \mathrm{mAb}$ ) treatments were conducted 5 days prior to initiation of the experiment. The treatment groups included DTX (10 mg/kg, Q3D, I.P.) combined with anti-PD-1 mAb (10 $\mathrm{mg} / \mathrm{kg}, \mathrm{Q} 3 \mathrm{D}$, I.P.), DTX (10 mg/kg, Q3D, I.P.) combined with anti-PD-L1 mAb (10 mg/kg, Q3D, I.P.), or negative control (vehicle, Q3D, I.P.). (B) Tumor growth monitored by BLI. (C) Tumors dissected from the SQCLC models after treatment. (D) Tumor growth curves during treatment, monitored using vernier calipers. (E) Tumor weight after treatment. (F) Intestinal gut microbiota diversity after treatment. (G) Microbial composition after treatment. Data are presented as mean $\pm S D$. N.S., no significance, ${ }^{*} p<0.05$; ${ }^{\star *} \mathrm{p}<0.01 ;{ }^{* *} \mathrm{p}<0.001$. BLI, bioluminescence imaging; DTX, docetaxel; Luc, luciferase; mAb, monoclonal antibody; PD-1, programmed death 1; PD-L1, programmed death-ligand 1; SQCLC, squamous cell lung carcinoma. 
which were consistent with the data obtained from animal models. On the other hand, given the survival benefits of combining pembrolizumab and concurrent MTD chemotherapy (carboplatin plus PTX or nab-PTX) in patients with SQCLC, we concluded that the chemotherapy dosages currently used for patients with cancer are functional, but not optimal. Additionally, it should be noted that long chemotherapy-free break periods are usually required to avoid systemic toxicity during MTD chemotherapy. In this scenario, even though the TIME can be activated to a certain extent, continual stimulation cannot be achieved, and reversal might occur during the drugfree periods. Further, in one of latest multicohort phase 2 clinical trials, Voorwerk et al demonstrated that 2-week low-dose chemotherapy induction followed by nivolumab contributed to an increased objective response rate in patients suffering from metastatic triple-negative breast cancer. ${ }^{39}$

With regard to the potential synergistic mechanisms between upfront metronomic DTX and anti-PD-1 mAb, we observed that macrophages, $\mathrm{CD} 8^{+} \mathrm{T}$ cells, and the gut microbiota might be involved. It has been reported that gut microbiota is the key orchestrator of immunity and bone marrow hematopoiesis, and are involved in the therapeutic responses to chemotherapy and immunotherapy ${ }^{40}{ }^{41}$ For instance, studies in mice have shown that the antitumor effects of platinum compounds were dramatically impaired when gut commensals were depleted by treatment with broad-spectrum antibiotics. ${ }^{42}$ Similar decreases in antitumor effects were also reported for anti-CTLA-4 mAb and anti-PD-L1 mAb therapy in antibiotic-treated mice. ${ }^{43}{ }^{44}$ In this study, the abnormal microbial composition and decreased diversity of gut microbiota were more severe after treatment with MTD DTX combined with anti-PD-1 mAb when compared with metronomic DTX plus anti-PD-1 mAb treatment, suggesting that the former might disturb the inherent balance of gut microbiota and reduce tumor inhibition effects. The underlying mechanisms through which gut microbiota may influence treatment effects require further exploration. Last but not least, numerous factors may affect the efficacy of chemoimmunotherapy. The drug dosage, sequence, and frequency of administration, as well as the time intervals between initiating chemotherapy and immunotherapy may all influence clinical benefits. Other possible variables should be the focus of future studies in order to further characterize and optimize chemoimmunotherapy for patients with SQCLC.

\section{CONCLUSIONS}

After stepwise investigation in SQCLC cell lines, syngeneic murine models, and patient samples, we concluded that low-dose chemotherapy contributed to remodeling a more active SQCLC-immune microenvironment compared with routine MTD chemotherapy. Upfront metronomic chemotherapy performed better with subsequent anti-PD-1/PD-L1 mAb treatment when compared with chemotherapy at the MTD. In contrast, the effects of MTD chemotherapy appeared to be more additive than synergic when combined with ICIs. Therefore, this preclinical study provides evidence for a novel strategy for the optimization of chemoimmunotherapy in patients with SQCLC. It is worth further exploring and then translating this approach into the clinic in the future.

\section{Author affiliations}

${ }^{1}$ Department of Medical Oncology, National Cancer Center/National Clinical Research Center for Cancer/Cancer Hospital, Chinese Academy of Medical Sciences \& Peking Union Medical College, Beijing, China

${ }^{2}$ CAS Key Laboratory of Molecular Imaging, the State Key Laboratory of Management and Control for Complex Systems, Institute of Automation, University of Chinese Academy of Sciences, Beijing, China

${ }^{3}$ University of Chinese Academy of Sciences, Beijing, China

${ }^{4}$ Department of Thoracic/Head and Neck Medical Oncology, UT MD Anderson Cancer Center, Houston, Texas, USA

${ }^{5}$ Beijing Advanced Innovation Center for Big Data-Based Precision Medicine, School of Medicine, Beihang University, Beijing, China

Contributors JW, JT, and HB designed the whole project and revised the manuscript. XH and YD carried out experiments, acquired and analyzed data, and wrote the manuscript. JW, XW, JD, RW, JX, PZ, DW, YT, JH, and KF gave technical, or material support and helped to collect patients' specimens.

Funding This study has received funding by Ministry of Science and Technology of China (No. 2017YFA0205); Ministry of Education Innovation Team Development Project (No. IRT-17R10); National Natural Science Foundation of China (No. 81871514, 81227901, 81470083, 91859119); National Natural Sciences Foundation Key Program (No. 81630071); National Key Research and Development Project (No. 2019YFC1315700); CAMS Innovation Fund for Medical Sciences (No. CIFMS 2016-I2M-3-008); CAMS Key Lab of Translational Research on Lung Cancer (No. 2018PT31035); Aiyou Foundation (No. KY201701).

Competing interests None declared.

Patient consent for publication Not required.

Ethics approval All the procedures involving DBA-2J mice were in accordance with the Institutional Animal Care and Use Committee (Permit Number 20170042). Regarding the patients' treatment and peripheral blood collection, all study participants provided informed consent, and the study design was approved by the appropriate ethics review board in National Clinical Research Center for Cancer/ Cancer Hospital (Permit Number NCC2018-074).

Provenance and peer review Not commissioned; externally peer reviewed.

Data availability statement All data relevant to the study are included in the article or uploaded as supplemental information. All data involving this study are available in the published article and supplemental information.

Supplemental material This content has been supplied by the author(s). It has not been vetted by BMJ Publishing Group Limited (BMJ) and may not have been peer-reviewed. Any opinions or recommendations discussed are solely those of the author(s) and are not endorsed by BMJ. BMJ disclaims all liability and responsibility arising from any reliance placed on the content. Where the content includes any translated material, BMJ does not warrant the accuracy and reliability of the translations (including but not limited to local regulations, clinical guidelines, terminology, drug names and drug dosages), and is not responsible for any error and/or omissions arising from translation and adaptation or otherwise.

Open access This is an open access article distributed in accordance with the Creative Commons Attribution Non Commercial (CC BY-NC 4.0) license, which permits others to distribute, remix, adapt, build upon this work non-commercially, and license their derivative works on different terms, provided the original work is properly cited, appropriate credit is given, any changes made indicated, and the use is non-commercial. See http://creativecommons.org/licenses/by-nc/4.0/.

ORCID iD

Jie Wang http://orcid.org/0000-0002-5602-0487 


\section{REFERENCES}

1 Bertaglia V, Vallone S, Pacchiana MV, et al. Advanced squamous lung cancer: therapeutic options, future directions, unmet needs and results of a monocentric survey. Lung Cancer Manag 2017;6:93-107.

2 Bunn PA. Karnofsky Award 2016: a lung cancer journey, 1973 to 2016. J Clin Oncol 2017;35:243-52.

3 Schiller $\mathrm{JH}$, Harrington D, Belani CP, et al. Comparison of four chemotherapy regimens for advanced non-small-cell lung cancer. $N$ Engl J Med 2002;346:92-8.

4 Cheng $\mathrm{H}$, Shcherba M, Kandavelou K, et al. Emerging drugs for squamous cell lung cancer. Expert Opin Emerg Drugs 2015;20:149-60.

5 Lazzari C, Karachaliou N, Gregorc V, et al. Second-Line therapy of squamous non-small cell lung cancer: an evolving landscape. Expert Rev Respir Med 2017;11:469-79.

6 Fritz JM, Lenardo MJ. Development of immune checkpoint therapy for cancer. J Exp Med 2019;216:1244-54.

7 Heinhuis KM, Ros W, Kok M, et al. Enhancing antitumor response by combining immune checkpoint inhibitors with chemotherapy in solid tumors. Ann Oncol 2019;30:219-35.

8 Wilky BA. Immune checkpoint inhibitors: the linchpins of modern immunotherapy. Immunol Rev 2019;290:6-23.

9 Paz-Ares L, Luft A, Vicente D, et al. Pembrolizumab plus chemotherapy for squamous non-small-cell lung cancer. $N$ Engl J Med 2018;379:2040-51.

10 Jotte RM, Cappuzzo F, Vynnychenko I, et al. IMpower131: primary pfs and safety analysis of a randomized phase III study of atezolizumab + carboplatin + paclitaxel or nab-paclitaxel vs carboplatin + nab-paclitaxel as $1 \mathrm{~L}$ therapy in advanced squamous NSCLC. JCO 2018;36:LBA9000.

11 Peters S, Reck M, Smit EF, et al. How to make the best use of immunotherapy as first-line treatment of advanced/metastatic nonsmall-cell lung cancer. Ann Oncol 2019;30:884-96.

12 Mathios D, Kim JE, Mangraviti A, et al. Anti-Pd-1 antitumor immunity is enhanced by local and abrogated by systemic chemotherapy in GBM. Sci Transl Med 2016;8:370ra180.

13 Galluzzi L, Buqué A, Kepp O, et al. Immunological effects of conventional chemotherapy and targeted anticancer agents. Cancer Cell 2015;28:690-714.

14 Siegel RL, Miller KD, Jemal A. Cancer statistics, 2019. CA A Cancer J Clin 2019;69:7-34.

15 Shafique M, Tanvetyanon T. Immunotherapy alone or chemoimmunotherapy as front-line treatment for advanced non-small cell lung cancer. Expert Opin Biol Ther 2019;19:225-32.

16 Nosaki K, Saka H, Hosomi Y, et al. Safety and efficacy of pembrolizumab monotherapy in elderly patients with PD-L1-positive advanced non-small-cell lung cancer: pooled analysis from the KEYNOTE-010, KEYNOTE-024, and KEYNOTE-042 studies. Lung Cancer 2019;135:188-95.

17 Okita R, Yukawa T, Nojima Y, et al. Mhc class I chain-related molecule $A$ and $B$ expression is upregulated by cisplatin and associated with good prognosis in patients with non-small cell lung cancer. Cancer Immunol Immunother 2016;65:499-509.

18 Zheng H, Zeltsman M, Zauderer MG, et al. ChemotherapyInduced immunomodulation in non-small-cell lung cancer: a rationale for combination chemoimmunotherapy. Immunotherapy 2017;9:913-27.

19 de Biasi AR, Villena-Vargas J, Adusumilli PS. Cisplatin-Induced antitumor immunomodulation: a review of preclinical and clinical evidence. Clin Cancer Res 2014;20:5384-91.

20 Cullis J, Siolas D, Avanzi A, et al. Macropinocytosis of nab-paclitaxel drives macrophage activation in pancreatic cancer. Cancer Immunol Res 2017;5:182-90.

21 Liu WM, Fowler DW, Smith P, et al. Pre-Treatment with chemotherapy can enhance the antigenicity and immunogenicity of tumours by promoting adaptive immune responses. $\mathrm{Br} \mathrm{J}$ Cancer 2010;102:115-23.
22 Galluzzi L, Humeau J, Buqué A, et al. Immunostimulation with chemotherapy in the era of immune checkpoint inhibitors. Nat Rev Clin Oncol 2020:bbaa184.

23 Pfirschke C, Engblom C, Rickelt S, et al. Immunogenic chemotherapy sensitizes tumors to checkpoint blockade therapy. Immunity 2016;44:343-54.

24 Song W, Shen L, Wang Y, et al. Synergistic and low adverse effect cancer immunotherapy by immunogenic chemotherapy and locally expressed PD-L1 trap. Nat Commun 2018;9:2237.

25 Weir GM, Hrytsenko O, Stanford MM, et al. Metronomic cyclophosphamide enhances HPV16E7 peptide vaccine induced antigen-specific and cytotoxic T-cell mediated antitumor immune response. Oncoimmunology 2014;3:e953407.

26 Denies S, Cicchelero L, Van Audenhove I, et al. Combination of interleukin-12 gene therapy, metronomic cyclophosphamide and DNA cancer vaccination directs all arms of the immune system towards tumor eradication. J Control Release 2014;187:175-82.

27 Liu X, Pu Y, Cron K, et al. Cd47 blockade triggers T cell-mediated destruction of immunogenic tumors. Nat Med 2015;21:1209-15.

28 Galon J, Bruni D. Approaches to treat immune hot, altered and cold tumours with combination immunotherapies. Nat Rev Drug Discov 2019;18:197-218.

29 Serrano-Del Valle A, Anel A, Naval J, et al. Immunogenic cell death and immunotherapy of multiple myeloma. Front Cell Dev Biol 2019;7:50.

30 Caamaño J, Hunter CA. Nf-kappaB family of transcription factors: central regulators of innate and adaptive immune functions. Clin Microbiol Rev 2002;15:414-29.

31 Jin X, Ding D, Yan Y, et al. Phosphorylated Rb promotes cancer immunity by inhibiting NF- $\mathrm{KB}$ activation and PD-L1 expression. $\mathrm{Mol}$ Cell 2019;73:22-35. e26.

32 Ramakrishnan SK, Zhang H, Ma X, et al. Intestinal non-canonical $\mathrm{NF} \kappa \mathrm{B}$ signaling shapes the local and systemic immune response. Nat Commun 2019;10:660.

33 Constantino J, Gomes C, Falcão A, et al. Dendritic cell-based immunotherapy: a basic review and recent advances. Immunol Res 2017;65:798-810.

34 Pandolfi F, Altamura S, Frosali S, et al. Key role of dAMP in inflammation, cancer, and tissue repair. Clin Ther 2016;38:1017-28.

35 Reagan-Shaw S, Nihal M, Ahmad N. Dose translation from animal to human studies revisited. Faseb J 2008;22:659-61.

36 Laksono BM, de Vries RD, Verburgh RJ, et al. Studies into the mechanism of Measles-associated immune suppression during a measles outbreak in the Netherlands. Nat Commun 2018;9:4944.

37 Gridelli C, Gallo C, Di Maio M, et al. A randomised clinical trial of two docetaxel regimens (Weekly vs 3 week) in the second-line treatment of non-small-cell lung cancer. the distal 01 study. Br J Cancer 2004;91:1996-2004.

38 Di Maio M, Perrone F, Chiodini P, et al. Individual patient data metaanalysis of docetaxel administered once every 3 weeks compared with once every week second-line treatment of advanced non-smallcell lung cancer. J Clin Oncol 2007;25:1377-82.

39 Voorwerk L, Slagter M, Horlings HM, et al. Immune induction strategies in metastatic triple-negative breast cancer to enhance the sensitivity to PD-1 blockade: the tonic trial. Nat Med 2019;25:920-8.

40 Roy S, Trinchieri G. Microbiota: a key orchestrator of cancer therapy. Nat Rev Cancer 2017;17:271-85.

41 Dzutsev A, Goldszmid RS, Viaud S, et al. The role of the microbiota in inflammation, carcinogenesis, and cancer therapy. Eur $\mathrm{J}$ Immunol 2015;45:17-31.

42 lida N, Dzutsev A, Stewart CA, et al. Commensal bacteria control cancer response to therapy by modulating the tumor microenvironment. Science 2013;342:967-70.

43 Vétizou M, Pitt JM, Daillère R, et al. Anticancer immunotherapy by CTLA-4 blockade relies on the gut microbiota. Science 2015;350:1079-84.

44 Sivan A, Corrales L, Hubert N, et al. Commensal Bifidobacterium promotes antitumor immunity and facilitates anti-PD-L1 efficacy. Science 2015;350:1084-9. 